\title{
The Joint Use of Sequence Features Combination and Modified Weighted SVM for Improving Daily Activity Recognition
}

\author{
Bilal M'hamed Abidine' ${ }^{1}$, Lamya Fergani ${ }^{1}$, Belkacem Fergani ${ }^{1}$ and Mourad Oussalah $^{2,3}$ \\ 'Laboratoire d'Ingénierie des Systèmes Intelligents et Communicants, LISIC Lab., Electronics and Computer Sciences Dept., \\ University of Science and Technology Houari Boumediene (USTHB), 32, El Alia, Bab Ezzouar, 16111 Algiers, Algeria \\ ${ }^{2}$ University of Birmingham, Electrical and Computer Engineering Dept., Birmingham, UK \\ ${ }^{3}$ University of Oulu, Centre for Ubiquitous Computing, Oulu 90014, Finland \\ b.fergani@usthb.dz, mbidine@usthb.dz, mourad.oussalah@oulu.fi;
}

\begin{abstract}
Two serious problems affecting the implementation of Human Activity Recognition (HAR) algorithms have been acknowledged. The first one corresponds to non-informative sequence features. The second is the class imbalance in the training data due to the fact that people do not spend the same amount of time on the different activities. To address these issues, we propose a new scheme based on a combination of Principal Component Analysis (PCA), Linear Discriminant Analysis (LDA) and the modified Weighted Support Vector Machines (mWSVM). First we added the most significant principal components to the set of features extracted using LDA.

This work shown that a suitable sequence feature set combined with the modified WSVM based our criterion classifier achieve good improvement and efficiency over the traditional used methods.
\end{abstract}

Keywords Activity Recognition - PCA - LDA • SVM . Imbalanced data classification

\section{Introduction}

Human Activity Recognition (HAR) is one of the most important tasks in pervasive computing applications [1-4] because of its contribution to several related areas, e.g., surveillance-based security, context aware computing and ambient assisted living [5]. It is aimed to determine the user's Activity Daily Living (ADL) such as cooking, brushing, sleeping, toileting and so on, using a set of fixed and wearable sensors readings. The ability to distinguish such activities is an essential step towards achieving activity-aware applications for real world settings. Advances and maturity in pervasive sensing technologies, microelectronics and (wireless) communication systems contributed largely to the development of automatic systems performing recognition of ADL tasks though development of cost and energy effective sensors. Strictly speaking, two types of sensors can be distinguished for ADL recognition task. The first relies on the use of wearable sensors (placed on a human body) to measure position, velocity and force [5]. Examples of activities that can be recognized using wearable sensors include walking, running and scrubbing. This is because such activities subsume mainly repetitive motions of human body, which are therefore largely constrained by the structure of the body. In contrast, other ADLs, e.g., toileting, sleeping and cooking are more appropriately recognized using external sensors placed at chosen locations at home or at target objects that the user is supposed to interact with (e.g., washing machine, stove, faucet, doors). For instance, a simple door switch sensor placed in a toilet would help to identify individual's toileting task. Similarly, a switch sensor placed in a bed can strongly trigger a sleeping task, and an activation of a cooking like appliances would suggest a cooking task, etc. Such binary (external) sensors are common in smart home projects [5-8] and rely widely on technology based of passive infrared or motion detectors. For example, in the MARC home, a set of (simple) sensors related to temperature on stove, mat, and cabinet doors placed in a kitchen have been used to detect meal preparation activities [9]. Other major smart home prototypes include Georgia Tech Aware Home project [6], CASAS project [10], MIT Lab [4], European Opportunity project [11], Mobile Data Challenge (led by Nokia) [12], Aware Home [13] and DOMUS [14].

Loosely speaking, homes and their appliances have highly variable layouts, and individuals perform activities in many different ways. A person may, for instance, take his lunch while watching television or setting in a kitchen or in a standing up position, etc. This results in a significantly distinct sensor activation profile based on the habits / routines of the occupant and the layout of a particular home [5]. This renders the task of automatic recognition of ADL very challenging and sometimes open to debate, which motivates the increasing work in the field during the last two decades as suggested by the review papers [5, 15-17]. On the other hand, the emerging trend of benchmarking initiatives such as the Opportunity Activity Recognition Challenge [11] and the Nokia Mobile Data Challenge [12] that make use of real-time sensory 
information is bringing a new maturity to the field of ADL recognition and allow researchers to build on the state of the art research with comparative analysis, an issue that will be explored in this current study. Several other challenges have also been pointed out by the ADL research community [5, 15]. This includes (i) the choice of sensors and their locations in the environment; (ii) the collection of data in realistic condition with flexibility to support new users; (iii) the selection (or extraction) of the attributes to be measured and processed; (iv) the design of the inference system that outputs the corresponding ADLs. With the use of benchmarking dataset, only the third and the fourth challenges are concerned in this paper.

From the inference system perspective, one distinguishes two main streams of approaches for ADL recognition task in home setting. The first one advocates a knowledge-driven approach where the relationship between sensor features and activities follows a descriptive approach, e.g., using an ontology where activities are recognized through their linking to constraints on sensor events $[15,18]$. The rationale is to exploit the logical knowledge representation for activity and sensor data modeling, and to use a logical reasoning to perform activity recognition. The second stream promotes a data-driven approach relying on machine learning and data mining techniques [19] to infer the underlying task where either supervised or unsupervised learning strategies are employed. The former is based on labeled data upon which an algorithm is trained, requiring increased computational power, while unsupervised strategy attempts to directly construct recognition models based on unlabeled data. In this way, as for any pattern recognition task, the keys to successful activity recognition are first to appropriately design a feature extraction strategy from the sensory data, and, second, to design a suitable classifier that infers the user's activity and provides an interpretation of the observed sensor patterns. The latter commonly requires data labeling either for machine learning or evaluation purposes. For this purpose, annotation of data for classification task can be performed in many different ways, e.g., use of cameras [4], self-reporting approaches, monitoring the diary activity [2], among others. Several classification algorithms have been employed for ADL recognition tasks [1-4], e.g., Hidden Markov model [2, 20], conditional random fields [21], linear discriminant analysis [22], sparse representation [23], Bayes approach [4], neural network [24], Support vector machine [25] and its softmargin multiclass SVM extension [26]. Motivated by extensive work by Google researchers, one shall also mention the growing interest to Deep-Learning (DP) like approaches [27] that make use of multiple processing layers and/or multiple non-linear transformations since the last decade. However, its application to HAR is still in its infancy, and relates predominantly to finding appropriate feature that best recognizes individual activity. Ordonez and Roggen [28] suggested a convolutional and LSTM recurrent units to recognize multimodal wearable activities. Plotz et al. [29] advocate the use of autoencoder network.
Feature extraction step consists in identifying a lower dimension set of features from the original data input that would ultimately ease the subsequent reasoning and classification task. Previous work in feature extraction for ADL rather advocates a context dependent approach where feature set can be binary, categorical or continuous depending on the type of sensors, classifier constraints and computational requirements [5, 15-17]. For instance, the use of motion sensors and accelerometers allows for a wider spectrum of statistical and energy related features, e.g., autoregressive coefficients, signal-magnitude area, tilt angle, spectral entropy, pairwise correlation, first and second order statistics, among others [30]. While in smart home setting, the binary like features yielding binary time series dataset can also be transformed into energy like features or reduced dimension dataset using PCA or LDA transformations [31]. Authors in [29] questioned the use of the same features for all ADL and rather suggested the use of different sets of features for distinct activity recognition tasks.

Another problem affecting the performance of activity classification algorithms is the class imbalance problem [32]. This is due to the fact that sampling dataset is not evenly distributed among various classes, because of insufficient coverage of some classes, referred to as minority class (es) with respect to other majority (or dominant) class (es), which makes the classifier overwhelmed by the majority class (es) and ignores the minority class examples. For instance, in daily activity tasks, sleeping is generally done only once a day, while toileting is done several times a day. Consequently, any automated learning system may have difficulties in learning the concept related to the minority class (toileting). This trivially can result in a degradation of the performance of the automated activity classification algorithm (s).

Various research methods [33-37] have been put forward to address the class imbalance problem. These solutions can be categorized into two main streams: data resampling and algorithmic level. The former involves a modification of the initial training dataset by under-sampling and over-sampling, which, in turn, purposefully modifies the class distributions, such that a rough balance between majority and minority classes is achieved while the classifier algorithm is kept unchanged. On the other hand, the algorithmic-like-methods keep the initial class distribution unchanged and rather introduce changes in the classifier design where some weighting scheme is introduced. Such solutions include the cost-sensitive learning [37] introduced in the case of SVM classifier, yielding Weighted Support Vector Machines (WSVM). In our study, we have adopted a modified WSVM that accommodates a multi-class classifier [38], where distinct weights are assigned automatically to data in order to account for imbalanced classes.

In [30], the authors use a combination of the PCA and LDA set of features for the task of object detection on datasets from UCI repository [39]. They demonstrate that such a combination approach has certain potential, especially when applied for $\mathrm{C} 4.5$ decision tree learning. This paper put forward a new framework, to handle both the issue of feature 
extraction and the class imbalanced problem for ADL recognition tasks. It advocates the combined PCA-LDA as a new feature sequence and the multi-class modified Weighted SVM as the new classifier. To the best of our knowledge such strategy has never been previously proposed to the ADL domain.

Next, the evaluation of the proposal PCA-LDA-WSVM is quantified using the open datasets (benchmarking) of [40-42] where comparisons with the state of art ADL classification algorithms, namely Hidden Markov Model (HMM), Conditional Random Filed (CRF), standard SVM, modified WSVM (mWSVM) and alternative hybrid models such as PCA-mWSVM, LDA-mWSVM and PCA-LDA-HMM are performed. Especially, given the popularity of probabilistic models HMM and CRF in daily activity recognition [2,3], new hybridation scheme involving such algorithms were used, e.g., PCA-LDA-HMM.

The contributions of this paper are the following. First, a new discriminative features set (PC-LD) was proposed. Second, a new scheme using a modified WSVM and PC-LD features set. Third, a comparative study over several state of the art hybrid models PCA-WSVM, LDA-WSVM and PCA-LDA-HMM as well as distinct classifiers, e.g., standard SVM, CRF and HMM, has been carried out in order to demonstrate the feasibility and enhanced performances of the proposal using multiple real-world smart home datasets.

The remainder of this paper is organized as follows. Section 2 presents the PCA, LDA and the combined PCALDA feature space. Section 3 highlights the design of the inference system that makes use of PCA-LDA and mWSVM classifier. Section 4 presents the experimental setup and discusses the results acquired throughout a series of benchmark dataset constituted of highly imbalanced datasets under different metrics. Finally, conclusions and future work are drawn in Section 5.

\section{Feature extraction}

\subsection{Notation}

The raw sensors data are discretized in time slices of constant length $\Delta t$. We denote a sensor reading as $x_{t}^{i}$, indicating whether sensor $i$ is fired at least once between time $t$ and time $t+\Delta t$, with $x_{t}^{i} \in\{0,1\}\left(x_{t}^{i}=1\right.$ if sensor $i$ is fired at least once between time $t$ and time $t+\Delta t$, zero otherwise). In a house with $n$ sensors installed, we define a global binary observation vector $\vec{x}=\left(x_{t}^{1}, x_{t}^{2}, \ldots, x_{t}^{n}\right)^{T}$ that accounts for all sensors. An activity at time slice $t$ is denoted by $A_{t}$ with $A_{t} \in\{1, \ldots, N\}$, where $N$ stands for the total number of daily living activities.

\subsection{Principal Component features}

The PCA [41] is a statistical procedure that uses an orthogonal transformation to convert a set of observations of possibly correlated variables into a set of linearly unrelated variables referred to as Principal Components Features (PCF) such that the first principal component has the largest possible variance and where the PCF correspond to the eigenvectors of the covariance matrix. More specifically, let $l$ be the total number of instances in the learning database and $(\vec{x})_{j}(j=1$ to $l$ ) be the total number of global binary state vectors. Then, the PCA transform is computed by performing the eigenvector decomposition of the covariance matrix $S$ :

$$
\begin{aligned}
& S=\sum_{j=1}^{l}\left((\vec{x})_{j}-\mu\right)\left((\vec{x})_{j}-\mu\right)^{T}, \quad \mu=\frac{1}{l} \sum_{j=1}^{l}(\vec{x})_{j} \\
& K=\operatorname{Eig}(S)
\end{aligned}
$$

Next, the PCF are calculated in the following way. First, the eigenvectors $K$ of matrix $S$ are rearranged according to their eigenvalues $\lambda_{i}$ ( $i=1$ to $n$, with $\lambda_{1} \geq \lambda_{2} \geq \ldots \geq \lambda_{n}$ ). Then the minimum number of principal components is determined by the percentage of variation explained. The number of PCF achieving a certain cumulative percentage of variance is calculated; namely, one determines an integer $p(p<n)$ such that

$$
\frac{\sum_{j=1}^{p} \lambda_{j}}{\sum_{j=1}^{n} \lambda_{j}} \geq \gamma
$$

Where $\gamma$ is a threshold, often taken to be high, corresponding to the proportion of the total variation that is explained by the first p principal components. Ideally, one aims for achieving higher proportion using only very few principal components. Authors in [43-44] suggested to choose such threshold beyond $80 \%$. In our case, we set $\gamma=85 \%$, which fully agrees with [42]. Therefore, the eigenvectors corresponding to eigenvalues $\lambda_{1}, \ldots, \lambda_{p}$ constitute the PCF, while the projection matrix $\Gamma_{l}$ of the PCF forms the PCA transform so that any new datum $T$ (given as a state binary vector as in $\vec{x}$ ) can be projected onto the reduced (feature) space using

$$
\left(T_{1}\right)_{p \times 1}=\left(\Gamma_{1}\right)_{p \times n}(T)_{n \times 1} \quad \text { with } \quad \Gamma_{1}=K^{T}
$$

\subsection{Linear Discriminant Features}

Unlike PCA which handles the data as a whole regardless their associated classes, LDA explicitly attempts to model the difference between the various classes that maybe assigned to original dataset and finds the linear combination of features that best separates the classes. Typically, the goal of LDA is to maximize the between-class measure while minimizing the within-class measure. Let $\mathrm{C}_{i}$ be the class containing the state binary vectors $\vec{x}$ corresponding to the $i^{\text {th }}$ activity class. Then the Linear Discriminant Features (LDF) are performed in different phases. First, the mean of each class is determined as 


$$
\mu_{i}=\frac{1}{n_{i}} \sum_{j=1}^{n_{i}}(\vec{x})_{j}^{i}
$$

Where $n_{i}$ is the number of instances assigned to the $i$-th class

Second, the between-class scatter matrix $S_{B}$ and the withinclass scatter matrix $S_{W}$ are calculated as:

$$
\mathrm{S}_{B}=\sum_{i=1}^{N} n_{i}\left(\mu_{\mathrm{i}}-\mu\right)\left(\mu_{\mathrm{i}}-\mu\right)^{T},
$$

where $\mu$ stands for the mean vectors of all inputs, or, equivalently, the mean of $\mu_{i}$ of (5), i=1, N.

$S_{W}=\sum_{i=1}^{N} \sum_{j=1}^{n_{i}}\left((\vec{x})_{j}^{i}-\mu_{i}\right)\left((\vec{x})_{j}^{i}-\mu_{i}\right)^{T}$

Third, one solves the generalized eigenvalue problem for the matrix $S_{W}^{-1} S_{B}$; namely,

$$
L=\operatorname{Eig}\left(S_{W}^{-1} S_{B}\right)
$$

Especially, the eigenvectors matrix $L$ guarantees the ratio $\frac{\operatorname{det}\left(S_{B}\right)}{\operatorname{det}\left(S_{W}\right)}$ is maximized, which assumes that the matrix $S_{W}$ is nonsingular [31]. It was shown in [45] that there will be at most $N-1$ eigenvectors whose eigenvalues are real-valued, which can represent a massive reduction in the dimensionality of the problem. In our study, we initially set the number of selected features equal $\mathrm{N}-1$ where $N$ is the number of the class activities.

Besides, many of the eigenvalues of $S_{W}^{-1} S_{B}$ are very small, and can be ignored without reducing the discriminative information significantly (e.g., by choosing some threshold $\varepsilon$ close to zero, so that the associated eigenvector is ignored whenever its associated eigenvalue is smaller than $\varepsilon$ ). The obtained projection matrix $\Gamma_{2}$ of LDF can therefore be used as a linear discriminant transform to any new dataset $T$ such that its projection will be

$$
\left(T_{2}\right)_{q \times 1}=\left(\Gamma_{2}\right)_{q \times n}(T)_{n \times 1} \quad \text { with } \Gamma_{2}=L^{T}
$$

\subsection{Combined PC-LD features}

The idea of combining PC and LD features is not fully new and seems rather intuitive given the inherent complementarity of the interpretations of the PCA and LDA formalisms in the sense that PCA performs dimensionality reduction while preserving as much of the variance in the high dimensional space as possible. While, LDA attempts to enhance the separation between the various classes. Consequently, in the context of daily activity recognition, the components that are obtained by the PCA have no discrimination characteristic between data in different activity classes. LDA-based feature extraction, although using class information, also has a serious drawback due to its parametric nature. Namely, the rank of $S_{B}$ is at most the number of classes minus one. Hence, if we integrate PCA and LDA in a systematic way, they could complement each other and be benefited from each other. The problem of combining the two features PC and LD has been approached from different perspectives, and one shall mention two approaches here. First, from a feature selection perspective [31], the problem might boil down to deciding for each time increment, which among the PC and LD features would likely provide the best performance in terms of classification rate. This would require designing a scoring function that would quantify the contribution of each feature individually with respect to some global performance metric. Such approach has been employed for face image recognition, see, for instance [46] and references therein. Second, from a fusion feature perspective, a typical solution is to create an augmented feature space which contains both PC and LD features. In [47], the authors contrasted an approach based on appending the distance vectors obtained using PCA to that of LDA features with an approach that creates a new distance vector by averaging PCA and LDA associated distances. Similarly, Pichenizkiy et al. [30] applied both the PCA and LDA transforms to the same training database, and then the merged feature space is obtained by concatenating PC features with LD features for the task of object detection on datasets from UCI repository [37]. Our approach follows the same spirit as [30] where an augmented feature formed by concatenating PC and LD features is constructed. Namely, each datum is represented in $(p+q)$ feature space.

Especially, it is easy to see that the augmented feature space has at most $p+N-1$ attributes. On the other hand, it is always possible to adjust the number of components in PCA and LDA ( $p$ and $q$ values) using the threshold values $\varepsilon$ and/or $\gamma$ if a large discrepancy between the number of PCA features and the number of LDA features is found, although in our case such scenario (almost) does not hold because of the restricted number of activity recognitions.

\section{Inference System}

\subsection{System overview}

As pointed out in the introduction part of this paper, the core of our proposal strategy relies on the use of a weighted support vector machine classifier whose inputs are binary state vectors, indicating the configuration of the various sensors, projected onto both PCA and LDA transforms. The choice of SVM like classifier [48-51] in this context is motivated by previous works as shown in [52], see also, review papers [5, 15-17] and references therein, where SVM demonstrated more robustness and less sensitivity to class discrimination problem. A generic diagram is shown in Figure 1. Especially, the initial dataset is divided into training and test datasets. These two sets are transformed independently using PCA and LDA methods. Then, we added the most significant principal components (PCF) generated by PCA transform to the set of features extracted (LDF) using LDA.

The constructed PCA-LDA space is therefore used for learning and testing the multi-class Weighted SVM classifier using a new automated criterion for weighting the data. In order to evaluate the proposal, and referring to what ought to 
be a reference in the field where HMM classifier was often backed for its high performance levels [2,3], we also implemented the corresponding PCA-LDA-HMM method, whose detail will be described in the next section.

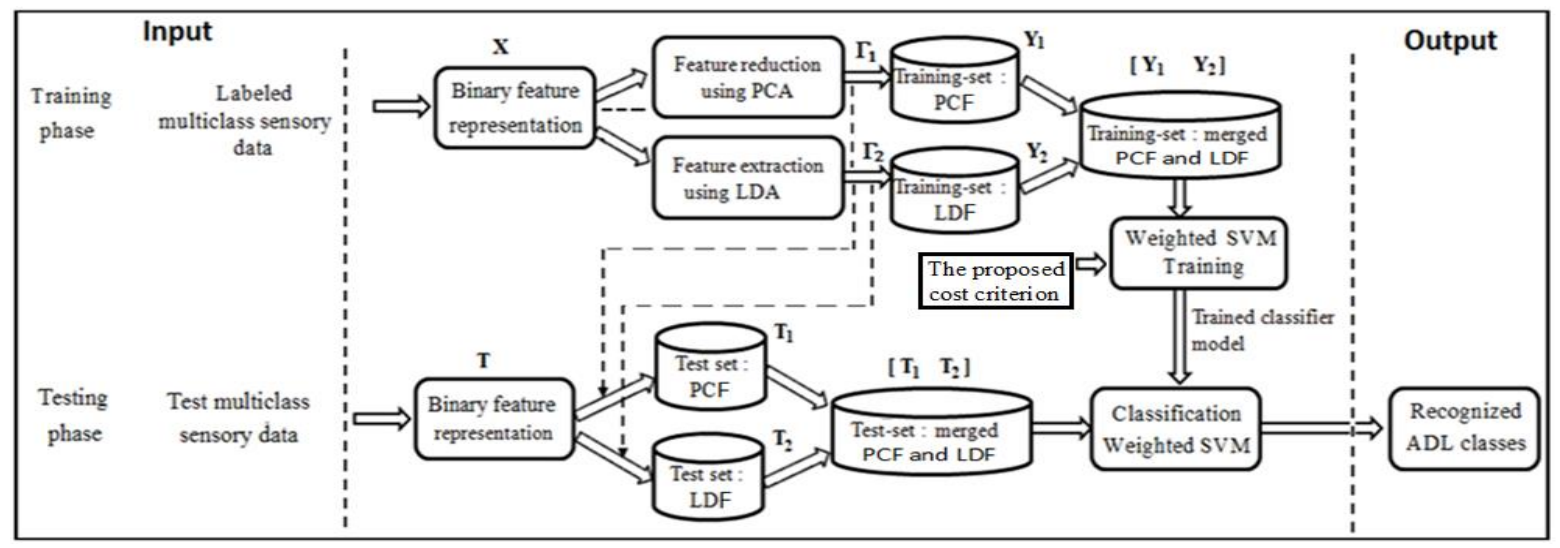

Fig. 1 Block diagram of the proposed activity recognition approach.

\subsection{Support Vector Machines}

SVM algorithm is adopted due to its proven performance elsewhere and its solid theoretical foundations [54]. SVM [4851] corresponds in its basic form to a two class classification problem. In order to preserve the coherence of the notations employed so far, let

$E=\left\{\left(y_{i}, d_{i}\right), y_{i} \in R^{p+q}, d_{i} \in\{+1,-1\}, i=1\right.$ to $\left.l\right\} \quad$ be $\quad$ the training dataset where the input $y_{i}$ (datum) corresponding to the $(p+q)$ feature vector, obtained using the combined PCA and LDA transforms, is labelled either belonging to the class $\left(d_{i}=+1\right)$ or not $\left(d_{i}=-1\right)$. The aim of SVM is to find the maximum-margin hyperplane that best separates the points $y_{i}$ whose associated $d_{i}=+1$ from those points whose $d_{i}=-1$. It is typical that such separation does not hold with original data points $y_{i}$ but rather through some non-linear transformation $\Phi$ of $y_{i}$ whose exact knowledge is not necessarily known, and one often limits to the knowledge of the associated kernel function. In this case, finding the hyperplane separating the positive and negative examples, quantified through the following equation

$$
s \bullet \Phi(y)+b=0
$$

boils down to the following optimization problem

$$
\begin{aligned}
& \min _{s, b} \frac{1}{2} s \bullet s \\
& \text { subject to } \quad d_{i}\left(s \bullet \Phi\left(y_{i}\right)+b\right) \geq 1,, i=1, \ldots, l
\end{aligned}
$$

where $s$ stands for the weight vector normal to the hyperplane that will be determined as part of the optimization problem (11). Therefore, the SVM decision function is

$$
f(y)=\operatorname{sign}(s \bullet \Phi(y)+b)
$$

Nevertheless, despite the use of higher dimensional feature space transformation $\Phi$, in practice, the classes still may not be separable. For this purpose, the soft-margin method introduced by Cortes and Vapnik [48] chooses a hyperplane that splits the examples as cleanly as possible, while maximizing the distance to the nearest cleanly split examples. The method introduces non-negative slack variables $\zeta_{i}$, which measure the degree of misclassification of the data $y_{i}$. The optimization problem of the soft-margin SVM is formulated as a tradeoff between a large margin and a small error penalty, where, as in [48], the same cost $C$ is assigned to both positive and negative misclassification as: $\min _{s, b, \zeta} \frac{1}{2} s \bullet s+C \sum_{i=1}^{l} \zeta_{i}$

subject to $d_{i}\left(s \bullet \Phi\left(y_{i}\right)+b\right) \geq 1-\zeta_{i}, \zeta_{i} \geq 0, i=1, \ldots, l$

Solving the formulation dual of SVM [49] gives a decision function in the original space for classifying a test point 


$$
\begin{aligned}
& y \in R^{p+q} \\
& f(y)=\operatorname{sign}\left(\sum_{i=1}^{m_{s v}} \alpha_{i} d_{i} K\left(y, y_{i}\right)+b\right)
\end{aligned}
$$

with $m_{s v}$ is the number of support vectors $y_{i} \in R^{p+q} . K(.,$. stands for the kernel function, which satisfies the Mercer's condition [48]. A typical kernel function commonly employed in this context is the Gaussian radial basis function. That is,

$$
\mathrm{K}\left(\mathrm{y}_{1}, \mathrm{y}_{2}\right)=\exp \left(-\left\|\mathrm{y}_{1}-\mathrm{y}_{2}\right\|^{2} / 2 \sigma^{2}\right)
$$

where $\sigma$ is the width parameter. In the construction of SVM, the misclassification cost parameter plays an indispensable role.

When the dataset is imbalanced, the density of the majority class examples would be higher than the density of minority class examples even around the class boundary region, where the ideal hyperplane would pass through. As a consequence, in order to reduce the total number of misclassifications in SVM learning, the predicted decision boundary can be shifted (or skewed) towards the minority class, see Fig. 2. This shift can cause the generation of more false negative predictions, which lowers the model's performance on the minority negative class. When the class imbalance is extreme, the SVM could produce models having largely skewed hyperplanes, which would even recognize all the examples as positives. This explains why SVM fails completely in situations of high degree of imbalance dataset, which motivates the so called Weighted Support Vector Machines (WSVM) detailed later on.

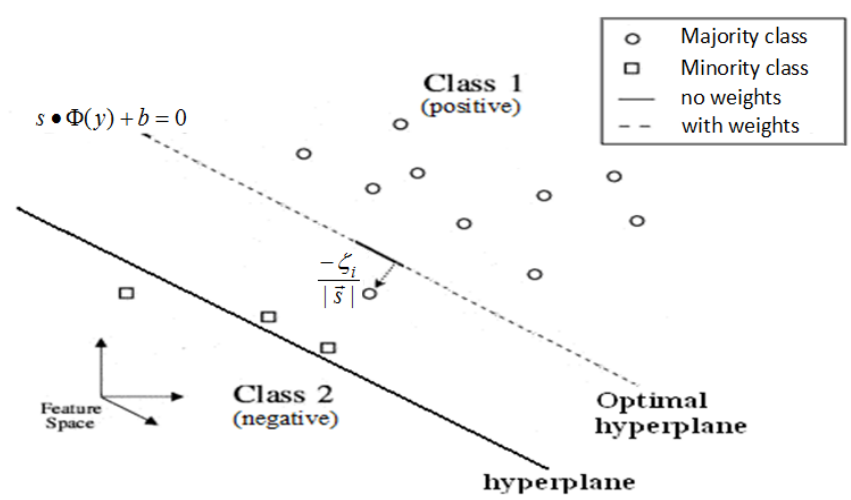

Fig. 2 Weighted SVM classification problem.

\subsection{Weighted Support Vector Machines (WSVM)}

Osuna et al [49] proposed a Weighted SVM algorithm to deal with the imbalanced dataset in the training phase by introducing two different cost parameter $C_{\text {- and }} C_{+}$in SVM optimization primal problem for the minority and majority classes, respectively, as given in expression (16). More formally, let $l_{+}$(resp. $l_{-}$) be the number of positive (resp. negative) instances in the initial database $\left(l_{-}+l_{+}=l\right)$. We assume positive class to be the majority class and negative class to be the minority class. The optimization problem of the SVM becomes:

$\min _{s, b, \zeta} \frac{1}{2} s \bullet s+C_{+} \sum_{i \mid d_{i}=1}^{l_{+}} \zeta_{i}+C_{-} \sum_{i \mid d_{i}=-1}^{l_{-}} \zeta_{i}$

subject to $d_{i}\left(s \bullet \Phi\left(y_{i}\right)+b\right) \geq 1-\zeta_{i}, \zeta_{i} \geq 0, i=1, \ldots, l$

The dual optimization problem of WSVM with different constraints on $\alpha_{i}$ can be solved similarly to [51]:

$$
\max _{\alpha_{i}}\left\{\sum_{i=1}^{l} \alpha_{i}-\frac{1}{2} \sum_{i=1}^{l} \sum_{j=1}^{l} \alpha_{i} \alpha_{j} d_{i} d_{j} K\left(y_{i}, y_{j}\right)\right\}
$$

subject to $0 \leq \alpha_{i}^{+} \leq C_{+}$, if $d_{i}=+1$, and

$$
\begin{aligned}
& 0 \leq \alpha_{i}^{-} \leq C_{-}, \quad \text { if } d_{i}=-1 \\
& \sum_{i=1}^{l} \alpha_{i} d_{i}=0, \quad i=1, \ldots, l
\end{aligned}
$$

Where $\alpha_{i}^{+}$and $\alpha_{i}^{-}$represent the Lagrangian multipliers of the positive and negative examples, respectively. This dual optimization problem can be solved in the same way as solving the normal SVM optimization problem. Solving the formulation dual of SVM [49] gives a decision function in the original space for classifying a test point $y \in R^{p+q}$ $f(y)=\operatorname{sign}\left(\sum_{i=1}^{m_{s v}} \alpha_{i} d_{i} K\left(y, y_{i}\right)+b\right)$

where $m_{s v}$ is the number of support vectors $y_{i} \in R^{p+q}$. A typical kernel function commonly employed in this context is the Gaussian radial basis function. That is,

$$
\mathrm{K}\left(\mathrm{y}_{1}, \mathrm{y}_{2}\right)=\exp \left(-\left\|\mathrm{y}_{1}-\mathrm{y}_{2}\right\|^{2} / 2 \sigma^{2}\right)
$$

where $\sigma$ is the width parameter. In the construction of WSVM, the misclassification cost parameter plays an important role.

Let $N_{B S V+}$ and $N_{B S V-}$ denote the number of boundary support vectors that belong to positive and negative classes, respectively. Similarly, let $N_{S V+}$ and $N_{S V}$ be the number of support vectors that belong to positive and negative classes, respectively. Then, using (18), it holds that:

$$
\sum_{i=1}^{l} \alpha_{i} d_{i}=\sum_{d_{i}=+1} \alpha_{i}-\sum_{d_{i}=-1} \alpha_{i}=0
$$

Because of the boundary support vector $\alpha_{i}=C$, when the number is $N_{B S V_{+}}$, the sum is always less than $\sum_{d_{i}=+1} \alpha_{i}$,

$$
N_{B S V+} C \leq \sum_{d i=+1} \alpha_{i}
$$

Moreover, because of the boundary support $\alpha_{i}$ is upper bounded by $C$, it holds that: 


$$
\sum_{d i=+1} \alpha_{i}, N_{S V+} C \geq \sum_{d_{i}=+1} \alpha_{i}
$$

Using (21-22), this entails:

$$
N_{B S V+} C \leq \sum_{d_{i}=+1} \alpha_{i} \leq N_{S V+} \cdot C
$$

Similarity, it holds:

$$
N_{B S V-} C \leq \sum_{d_{i=-1}} \alpha_{i} \leq N_{S V-} \cdot C
$$

Substituting $C$ by $C . w_{+}$in (24) and C.w in (25), and setting

$$
\sum_{d_{i}=+1} \alpha_{i}=\sum_{d_{i}=-1} \alpha_{i}=A,(24-25) \text { are equivalent to: }
$$$$
\begin{aligned}
\frac{N_{B S V+}}{l_{+}} & \leq \frac{A}{C \cdot w_{+} l_{+}} \leq \frac{N_{S V+}}{l_{+}} \\
\frac{N_{B S V-}}{l_{-}} & \leq \frac{A}{C \cdot w_{-} l_{-}} \leq \frac{N_{S V-}}{l_{-}}
\end{aligned}
$$

Huang et al. [35] put forward a WSVM whose coefficients are such that:

$$
\begin{aligned}
& C_{+}=C \times w_{+} \\
& C_{-}=C \times w_{-}
\end{aligned}
$$

In order to get the same error rate in both classes, let

$$
\frac{A}{C . w_{+} \cdot l_{+}}=\frac{A}{C \cdot w_{-} \cdot l_{-}}
$$

By introducing (28) and (29) in equation (30), we can get the following relation

$$
\frac{C_{+}}{C_{-}}=\frac{l_{-}}{l_{+}}
$$

where $w^{+}$and $w^{-}$are the weights assigned to positive and negative classes, respectively. Authors in [61] suggested the use of cross validation method in order to determine the value of the common cost parameter $C$ of the WSVM.

\subsection{Proposed modified multi-class WSVM}

Some authors [33, 37, 50] have proposed adjusting different cost parameters to cope with the minority class members' misclassification. Veropoulos et al. in [50] suggested to increase the cost of the minority class (i.e., $C_{-}>C_{+}$) to obtain a larger margin on the side of the smaller class. However no guidelines about the choice of cost factors were provided. To extend SVM to the multi-class scenario in order to deal with $N$ classes (daily activities), we used different cost misclassification $C_{i}$ per class [38]. The idea is to let the user set individual weights for individual training examples, which are then used in WSVM training. By taking $C_{-}=C_{i}$ and $C_{+}=C$, the main ratio cost value $C_{i}$ for each activity class can be obtained as:
$\frac{C_{+}}{C_{i}}=\frac{1_{i}}{1_{+}}$with $i=1, \ldots, N$

$C_{i}=\operatorname{round}\left(C \times\left[l_{+} / l_{i}\right]\right)$

[.] is integer part of the quantity under square bracket.

Notice that it always holds that $C_{i} \geq C$.

For training data from two classes $\mathrm{C}_{i}$ and $\mathrm{C}_{j}$, we can modify the objective function (16) to

$\min _{s, b, \zeta} \frac{1}{2} s \bullet s+C\left(\left[\frac{l_{+}}{l_{i}}\right] \sum_{i \mid d_{i}=1}^{l} \zeta_{i}+\left[\frac{l_{+}}{l_{j}}\right] \sum_{i \mid d_{i}=-1}^{l} \zeta_{i}\right)$

subject to $d_{i}\left(s \bullet \Phi\left(y_{i}\right)+b\right) \geq 1-\zeta_{i}, \zeta_{i} \geq 0, i=1, \ldots, l$

A three class classification problem is provided in [36]. The dual optimization problem of WSVM gives the same Lagrangian as in (17) with $C_{+}=C\left[l_{+} / l_{i}\right]$ and $C_{-}=C\left[l_{+} / l_{j}\right]$. That is, the WSVM algorithm would not tend to skew the separating hyperplane towards the minority activity class examples to reduce the total misclassifications as the minority class examples are now assigned with a higher misclassification cost. This criterion is motivated by the findings reported by Veropoulos et al. [50] where it stated that the tradeoff $C_{i}$ associated with the smallest class is large to improve the low classification accuracy caused by imbalanced samples.

In this study, a software package LIBSVM [55] was used to implement the multiclass classifier algorithm. The one-to-one strategy is adopted for this purpose. This method is better than one-versus-all schema because in addition to the naturally occurring class imbalance problem, the imbalanced data situation may also occur in one-against-all schema in multiclass classification. Specifically, the one-to-one method consists in constructing $N(N-1) / 2$ sub-classifiers where each one is trained on a subset of the training data from the two activity classes as shown in Figure 3. When all classifiers are constructed, a majority voting strategy is used. Each new example is sent for classification to all the classifiers and the decision that has the most important number of votes is the final taken decision. Chen et al. [56] discussed issues of using the same or different SVM parameters for the $N(N-1) / 2$ two-class problem. Their preliminary results showed that both approaches provide almost similar accuracy levels. 


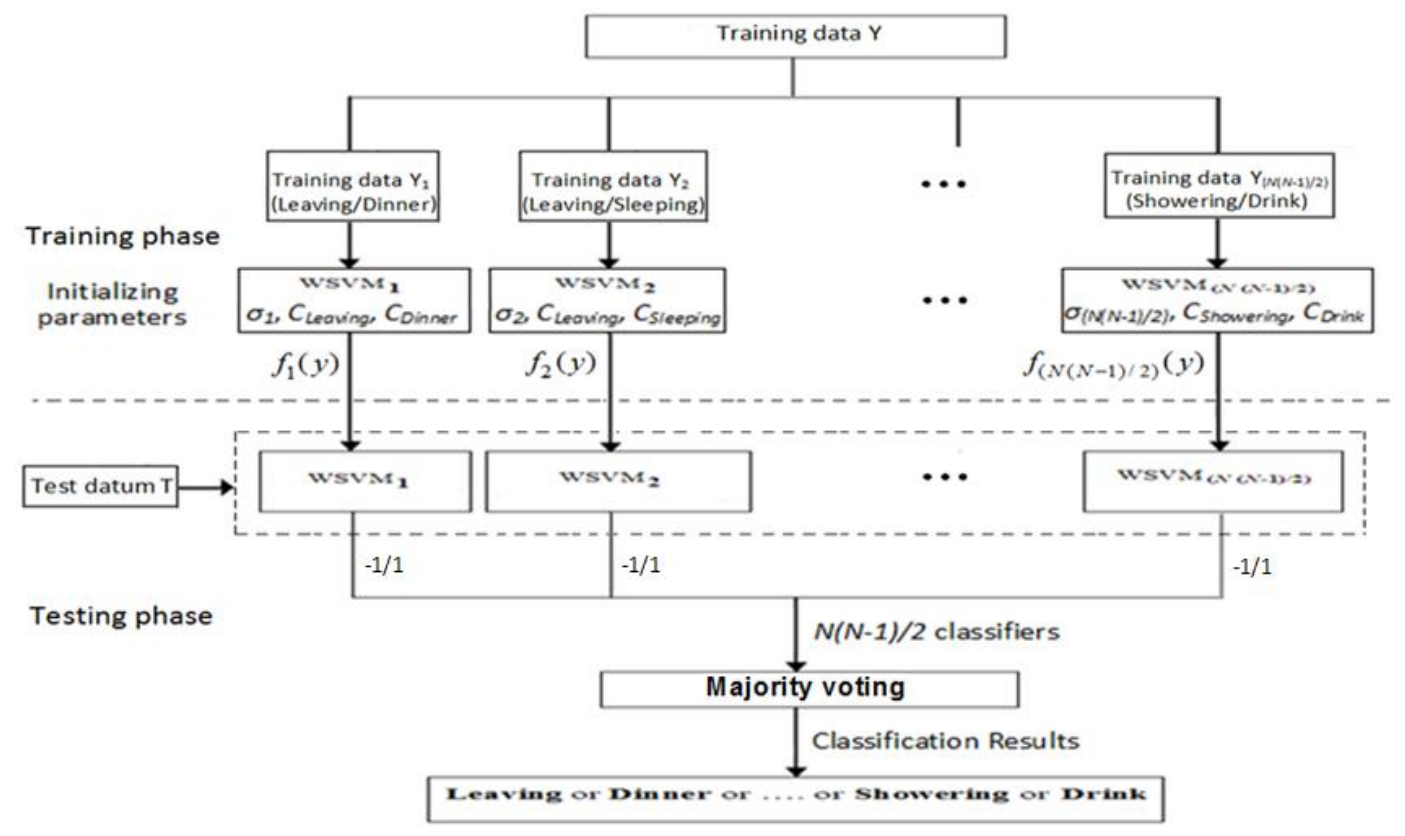

Fig. 3 Structure of Multi-class Weighted SVM using one-to-one approach.

The pseudo-code showing the execution of the suggested multi-class PCA-LDA-WSVM based our criterion is summarized in Table 1 , where $d_{i, j}$ denotes the decision given, for a new datum, by the modified WSVM trained to distinguish the classes $i$ and $j$. If $d_{i, j}=0.5$, we consider the classifier that has the highest margin.

Table 1 Summary of the proposed activity recognition method

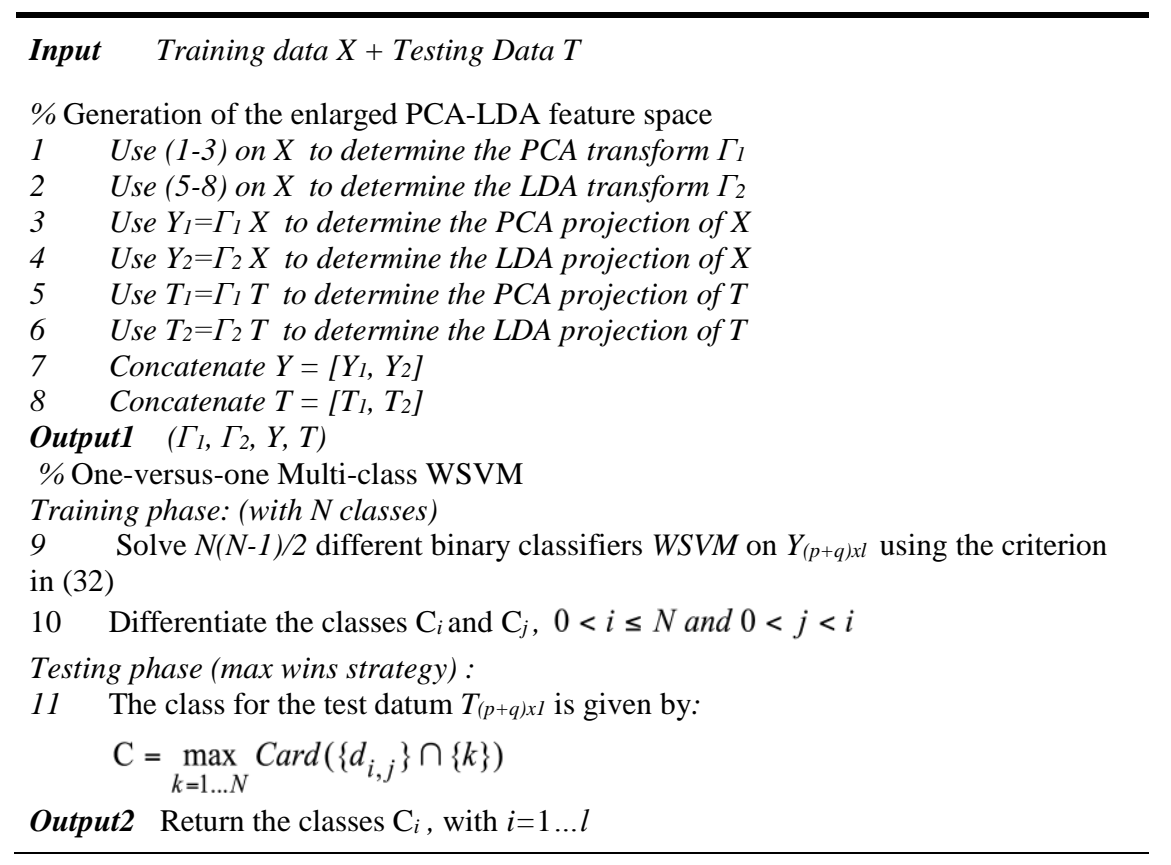

3.5 Alternative inference system model: Hidden Markov Model (HMM)
The Hidden Markov Model (HMM) is stressed in this study because it constituted an inevitable reference in ADL, especially, with respect to the employed benchmark datasets $[2,3]$. Therefore, a trivial question is whether the newly established augmented feature space works well with HMM 
instead of (modified) WSVM. To answer such question a hybridation PCA-LDA-HMM is investigated in this section. More specifically, a (probabilistic) sequential process consists of a hidden variable $y$ and an observable variable $x$ at each time step. In our case the hidden variable stands for one activity among the set of activities to recognize, and the observable variable is associated to the set of features in the sensor feature space. HMM is governed by two key assumptions which are highlighted in Fig. 4 and summarized in:

- The hidden variable at time $t$, namely $y_{t}$, depends only on the previous hidden variable $y_{t-1}$ (Markov assumption [53]).

- The observable variable at time $t$, namely $x_{\mathrm{t}}$, depends only on the hidden variable $y_{\mathrm{t}}$.

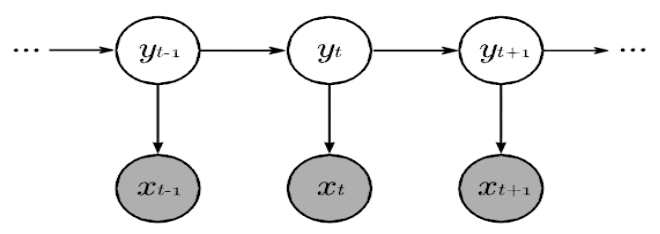

Fig. 4 Graphical representation of HMM. The shaded nodes represent observable variables, while the white nodes represent hidden ones.

With these assumptions we can specify an HMM using three probability distributions: the distribution over initial states $p\left(y_{1}\right)$ parameterized by $\pi$; the transition distribution $p\left(y_{t} \mid y_{t-1}\right)$, parameterized by $A$, represents the probability of going from one state to the next; and the observation distribution $p\left(x_{t} \mid y_{t}\right)$ parameterized by $B$, indicating the probability that the state $y_{t}$ would generate observation vector $x_{t}$.

The entire model is therefore parameterized by the aforementioned three parameters: $\theta=\{\pi, A, B\}$. Learning the parameters of these distributions corresponds to maximizing the joint probability $\mathrm{p}(\mathrm{x}, \mathrm{y})$ between the sensor data and activities in the training data; namely,

$$
P(\mathrm{x}, \mathrm{y})={ }_{t=1}^{T} p\left(y_{t} \mid y_{t}\right) p\left(x_{t} \mid y_{t}\right)
$$

We write the distribution over initial states $p\left(\mathrm{y}_{1}\right)$ as $p\left(\mathrm{y}_{1} \mid \mathrm{y}_{0}\right)$, to simplify notation.

It must be noted that, when using the HMM, each feature is modeled by an independent Bernoulli distribution, as proposed by previous studies $[2,3]$. Then, a global transition matrix $A$ with transition coefficients $\mathrm{a}_{i j}$ (the probabilities between different classes $i$ and $j$ from the time $t-1$ to $t$ ) is calculated. The transition coefficients can be estimated using an iterative forward-backward algorithm from HMM training dataset [53]. The inference problem for the HMM consists of finding the single best state sequence that maximizes $p(\mathrm{x}, \mathrm{y})$. Although the number of possible paths grows exponentially with the length of the sequence, we can efficiently find the sequence of activities that maximizes this probability using the commonly used Viterbi algorithm [53]. The summary of our implemented HMM method in both training and test phases can be summarized into the following.

i) The number of states $(\mathrm{N})$ and also the structure of transition matrix are fixed.

ii) The sequence of observation containing all of the training samples which belong to a specific class is calculated. The obtained samples are partitioned using Kmeans algorithm where the number of partitions is equal to the number of states.

iii) The initial values of mean and standard deviation corresponding to Gaussian model are set by K-means algorithm,

iv) HMM model for classification of activities is trained using the Baum-Welch algorithm [45].

v) The parameters of each model corresponding to each class are saved and used for the test phase.

vi) In the test phase, Forward-Backward algorithm is employed. Besides, by comparing the estimated log likelihood probabilities of test data, the class of each data is identified

\section{Experimental Results and Discussion}

In this section, we first give a description of the employed datasets, then the testing setup is detailed, and, finally, the results are highlighted and discussed.

\subsection{Datasets}

In this paper we have employed datasets generated by a set of simple state-change sensors installed in four different environments. Each dataset is constituted of a binary temporal dataset originated from a number of sensing nodes that monitored the ADLs performed in a home setting of a single resident. These selected activities are based on the Katz ADL (Activities of Daily Life) index, to assess the physical capabilities of elderly people [57], see Table 2. These datasets have been broadly employed in previous studies [1-3] and are publicly available for download from reference [40-42]. The layout of the different home settings differs strongly, as well as the sensors configuration. The type of sensors employed to monitor the users was chosen according to two main criteria: ease of installation and minimal intrusion. The installed sensors include passive infrared sensors to detect motion at specific areas; reed switches for open/close states of doors and cupboards, and float sensors to measure the toilet being flushed. The sensor data were collected through a base station and labelled according to the corresponding activity. An overview of the datasets can be found in Table 3. As previously mentioned, sensor data streams were segmented in fixed time slices of constant length. A list of activities that were annotated for all datasets together with the number of observations of each activity is described in Table 2. Any 
period of time at which no activity took place was labelled as 'Idle'.

Table 2 List of activities annotated for each house and the number of observations of each activity. Bold characters represent abbreviated activity names.

\begin{tabular}{|c|c|c|c|}
\hline TK26M & TK57M & Tap80F & OrdonezA \\
\hline $\begin{array}{l}\text { Idle(4627) }_{(426)} \\
\text { Leaving }_{(22617)} \\
\text { Toileting }_{(380)} \\
\text { Showering }_{(265)} \\
\text { Sleeping }_{(11601)} \\
\text { Breakfast(109) }_{(102)} \\
\text { Dinner }_{(348)} \\
\text { Drink}_{(59)}\end{array}$ & 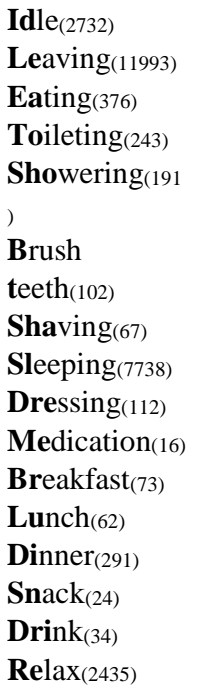 & 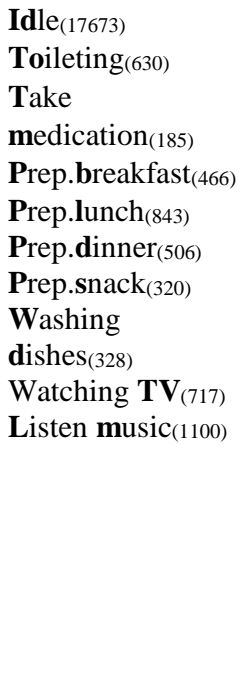 & $\begin{array}{l}\text { Idle }_{(1307)} \\
\text { Sleeping }_{(7886)} \\
\text { Toileting(}_{(173)} \\
\text { Showering }_{(121)} \\
\text { Breakfast }_{(132)} \\
\text { Grooming(154) }_{(154)} \\
\text { Spare_Time/ }_{-} \\
\text {TV }_{(8646)} \\
\text { Leaving }_{(1692)} \\
\text { Lunch }_{(331)} \\
\text { Snack }_{(14)}\end{array}$ \\
\hline
\end{tabular}

Table 3 The summary of four smart home datasets used in the evaluation of proposed approach.

\begin{tabular}{ccclc}
\hline Houses & TK26M & TK57M & Tap80F & OrdonezA \\
\hline Setting & Apartment & House & Apartment & House \\
Annotation & $\begin{array}{l}\text { Bluetooth } \\
\text { headset+ } \\
\text { Speech } \\
\text { recognition } \\
\text { software }\end{array}$ & $\begin{array}{l}\text { Hand- } \\
\text { written } \\
\text { Diary }\end{array}$ & PDA & $\begin{array}{l}\text { Hand-written } \\
\text { Diary }\end{array}$ \\
Duration & 28 days & 18 days & 14 days & 14 days \\
Sensors & 14 & 21 & 70 & 12 \\
Activities & 8 & 16 & 10 & 10 \\
\hline
\end{tabular}

\subsection{Data Analysis Setup}

Classification models were validated by splitting the original data into a test and training set using a " $k$-Leave One Day Out cross validation" approach, retaining one full day of sensor readings for testing and using the remaining sub-samples as training data. The process is then repeated for each day and the average performance measure is reported for $k$ days. This produces unbiased but high-variance error estimates. By doing so, the inputs are labelled by concatenating the results acquired for each test day. Using PCA and LDA projections, a vector of features was generated for each slice, which is then used as an input to WSVM classifier that outputs the daily activity class corresponding to the underlying dataset.

Sensors outputs are binary and contain one entry for each sensor; namely, a binary state 0 or 1 depending whether the sensor has been activated or not during the underlying time window. The raw sensor representation uses the sensor data in the same way it was received from the sensors network. We do not use this data representation as observations; instead we use the combination of "Change Point" and "Last" representation which has been shown to give much better results in activity recognition [2]. Typically, the "Change point" representation gives 1 when the sensor reading changes from 0 to 1 or from 1 to 0 , while the "Last sensor" representation continues to assign a 1 value to the last sensor that changed state until a new sensor changes state. Combining the feature representations was done by concatenating the feature matrices. These feature representations originally proposed by Kasteren et al. [2] are shown in Fig. 5.

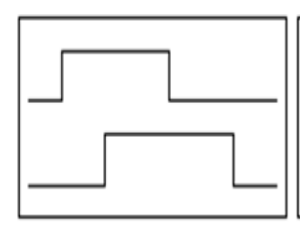

(a)

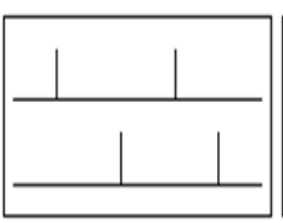

(b)

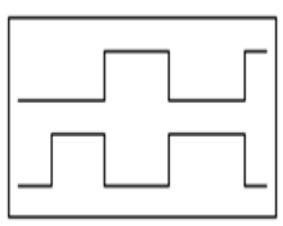

(c)
Fig. 5 Example of sensor firing showing the a) Raw, b) Change point and c) Last observation representation.

\subsection{Performance metrics}

Table 3 enables us to see clearly the disparity in terms both the frequency of occurrence as well as their associated durations (e.g. leaving and sleeping). Sleeping activity generally takes up considerably more time slices than the toileting activity. Therefore, the datasets suffer from a severe class imbalance problem due to the nature of the data. A high accuracy may represent the assignment of correct labels to the class with the majority of instances. In our case, rare classes are of interest; therefore we used a set of performance metrics to evaluate the performance of our designed inference system in conjunction to other state of art alternative approaches. This consists of the F-measure, which is calculated from the Precision and Recall scores. On the other, in order to evaluate the sensitivity of the classifiers in the multi-class classification problem, the notions of True Positive (TP), False Negatives (FN) and False Positives (FP) for each class separately, have also been implemented. These measures can be calculated using the confusion matrix shown in Table 4. 
Table 4 The Confusion Matrix. The $\epsilon_{\mathrm{ij}}$ terms show the error between true class $i$ and inferred class $j$.

\begin{tabular}{ccccc}
\hline & \multicolumn{3}{c}{ Inferred } & \\
\cline { 2 - 4 } True & 1 & 2 & 3 & FN \\
\hline 1 & $T P_{1}$ & $\epsilon_{12}$ & $\epsilon_{13}$ & $F N_{1}$ \\
2 & $\epsilon_{21}$ & $T P_{2}$ & $\epsilon_{23}$ & $F N_{2}$ \\
3 & $\epsilon_{31}$ & $\epsilon_{32}$ & $T P_{3}$ & $F N_{3}$ \\
\hline FP & $F P_{1}$ & $F P_{2}$ & $F P_{3}$ & Total \\
\hline
\end{tabular}

These measures are calculated as follows:

$$
\begin{aligned}
& \text { Accuracy }=\frac{\sum_{\mathrm{i}=1}^{\mathrm{N}} \mathrm{TP}_{\mathrm{i}}}{\text { Total }} \times 100 \% \\
& \text { Precision }=\frac{1}{N} \sum_{i=1}^{N}\left[\frac{\mathrm{TP}_{i}}{\mathrm{TP}_{i}+\mathrm{FP}_{i}}\right] \times 100 \% \\
& \text { Recall }=\frac{1}{N} \sum_{i=1}^{N} \frac{\mathrm{TP}_{i}}{\mathrm{TP}_{i}+\mathrm{FN}_{i}} \times 100 \% \\
& =\frac{1}{N} \sum_{i=1}^{N} \text { Accuracy }_{i} \times 100 \% \\
& \mathrm{~F}-\text { Measure }=\frac{2 . \text { Precision } \cdot \text { Recall }}{\text { Precision }+ \text { Recall }} \times 100 \%
\end{aligned}
$$
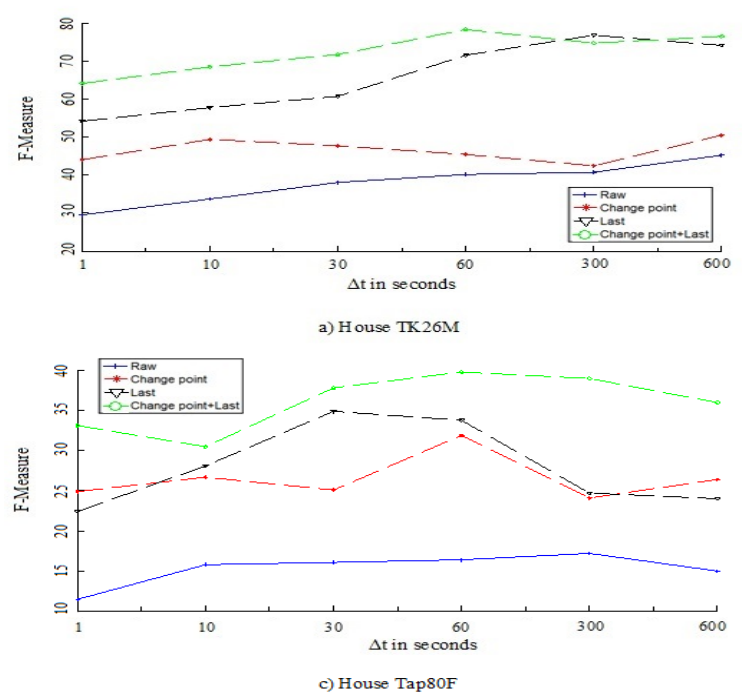

Fig. 6 F-Measure performance of the proposed method for the different houses using different time slices length to discretize the data.

The goal of the second experiment is to evaluate the performance of the proposed approach.

Next, in order to evaluate the performance of our proposed approach, a comparison with some state of art models has been carried out. This consists of HMM, CRF, SVM, WSVM, PCA-WSVM, LDA-WSVM and PCA-LDA-HMM. In PCA-
Measuring accuracy is a typical way of evaluating timeseries analysis. However, we also report the F-measure, which is a common technique in datasets with a dominant class. In this case classifying all the test data as the dominant class yields good accuracy, but no useful output. The F-measure, however, would remain low, and therefore be representative of the actual model performance.

\subsection{Results}

In the first experiment, we present our findings for determining the ideal time slice length for discretizing the sensor data. This Experiment was run using only our proposed method for the different datasets. We experimented using all the feature representations, to rule out any bias towards any of the representations. These feature representations were used standalone and combined. Combining the feature representations was done by concatenating the feature matrices. The F-measure values for the various time slice $[1 \mathrm{~s}$, $10 \mathrm{~s}, 30 \mathrm{~s}, 60 \mathrm{~s}, 300 \mathrm{~s}, 600 \mathrm{~s}]$ lengths are plotted in Figure 6. We see that for each house time slice length $\Delta t=60$ seconds achieves consistently the best performance for Change point+Last feature representation for all datasets. This interval length is considered long enough to be discriminative and short enough to provide good accuracy labelling results [2], since with larger time slices the shorter activities would not survive the discretization process. After segmentation, there were a total of 40006 time slices for TK26M dataset, 26489 time slices for TK57M dataset, 22768 time slices for Tap80F dataset, and 20456 time slices for OrdonezA dataset.
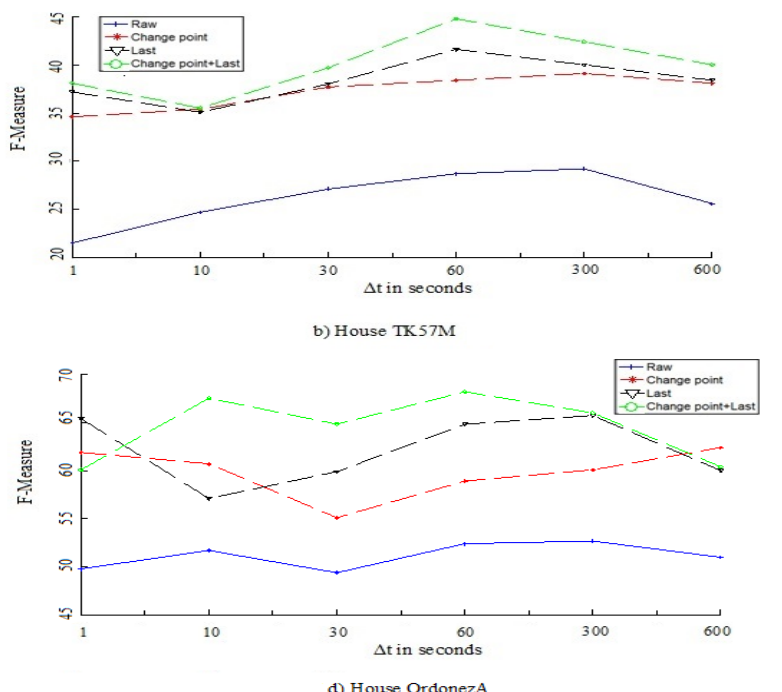

LDA-HMM, the part of WSVM in the PCA-LDA-WSVM system (in Fig. 1) is replaced by HMM. We used the concatenation of change point and last data representation "Changepoint+Last". We evaluated the performance of these methods on the imbalanced datasets of different houses in which majority class are all classes that have a longer duration 
(e.g. 'Idle', 'Leaving', 'Sleeping', 'Relax' for the TK26M and TK57M datasets; 'Idle' for Tap80F dataset and 'Idle', 'Sleeping', 'Spare_Time/TV', 'Leaving' for OrdonezA dataset), while others are the minority classes. These algorithms are tested under MATLAB environment, where LibSVM library [55] was used to implement our modified WSVM. Firstly, we optimized the number of clusters $K$ for HMM with $\mathrm{K}$-means clustering by maximizing the error rate of leave-one-day-out cross validation technique. Since we have no solid background about how many clusters are best for prediction, we vary the $\mathrm{K}$ parameter in our experiments in the range [1-300]. We get $\mathrm{K}=33,44,149$ and 61 for the TK26M, TK57M, Tap80F and OrdonezA datasets, respectively.

Then, we optimized the SVM hyper-parameters $(\sigma, C)$ for all training sets in the range [0.1-2] and $\{0.1,1,5,10,100\}$, respectively, to maximize the error rate of leave-one-day-out cross validation technique. The pairs $\left(\sigma_{o p t}, C_{o p t}\right)=(1.7,1)$, $\left(\sigma_{o p t}, C_{o p t}\right)=(2.0,1),\left(\sigma_{o p t}, C_{o p t}\right)=(1.2,1)$ and $\left(\sigma_{o p t}, C_{o p t}\right)=$ $(1.3,5)$ are found to be optimal the training datasets of TK26M, TK57M, Tap80F and OrdonezA, respectively. Finally, for WSVM classification method, we optimized locally the cost parameter $C_{i}$ adapted to different classes where the common cost parameter is fixed $C=1$. The weights assigned to various classes using expression (32) whose values for different dataset are given in Tables 5 to 8 . These values are given for the first iteration of leave one day out cross validation approach.

Table 5 Weights $w_{i}$ in TK26M dataset.

\begin{tabular}{ccccccccc}
\hline ADL & Id & Le & To & Sh & Sl & Br & Di & Dr \\
\hline$w_{i}$ & 5 & 1 & 61 & 88 & 2 & 216 & 73 & 419
\end{tabular}
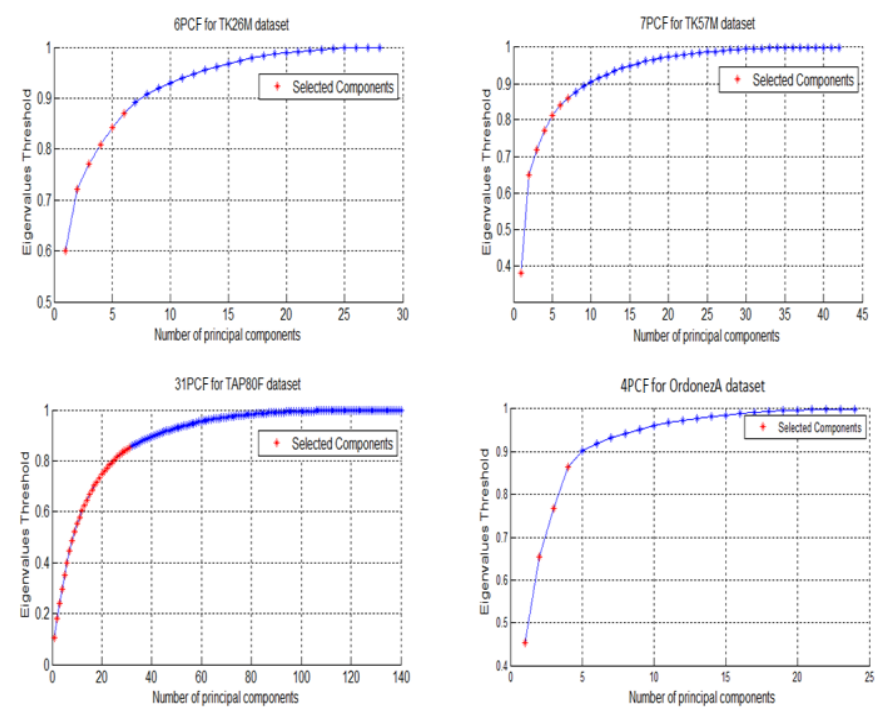

Fig. 7 Feature extraction by Principal Component Analysis (PCA).
Table 6 Weights $w_{i}$ in TK57M dataset.

\begin{tabular}{cccccccccc}
\hline ADL & Id & Le & Ea & To & Sho & B.t & Sha & Sl & Dre \\
\hline$w_{i}$ & 4 & 1 & 32 & 50 & 63 & 118 & 179 & 2 & 107 \\
\hline $\mathrm{ADL}$ & $\mathrm{Me}$ & $\mathrm{Br}$ & $\mathrm{Lu}$ & $\mathrm{Di}$ & $\mathrm{Sn}$ & $\mathrm{Dri}$ & $\mathrm{Re}$ & & \\
$w_{i}$ & 749 & 164 & 193 & 41 & 500 & 375 & 5 & &
\end{tabular}

Table 7 Weights $w_{i}$ in Tap80F dataset.

\begin{tabular}{ccccccccccc} 
ADL & Id & To & T.m & P.b & P.l & P.d & P.s & W.d & TV & L.m \\
\hline$w_{i}$ & 1 & 30 & 92 & 38 & 21 & 36 & 72 & 53 & 32 & 17 \\
\hline
\end{tabular}

Table 8 Weights $w_{i}$ in OrdonezA dataset.

\begin{tabular}{ccccccccccc}
\hline ADL & Id & Sl & To & Sh & Br & Gr & TV & Le & Lu & Sn \\
\hline$w_{i}$ & 6 & 1 & 49 & 71 & 66 & 53 & 1 & 5 & 27 & 610 \\
\hline
\end{tabular}

As it can be noticed from these tables, the minority classes are assigned larger value of $w_{i}$ as compared to the majority classes. Intuitively, this fact induces a classifier's bias in order to give more importance to the minority classes.

We reported in figures 7 and 8 the process of feature extraction in terms of number of PCF and LDF using two methods: PCA and LDA. The total number of features is 28, 42, 140 and 24 for TK26M, TK57M, Tap80F and OrdonezA datasets, respectively. This is due to the fact that we used in this work the concatenation of two matrices the "Changepoint" and "Last" representations. In order to stress the comparison between PCA and LDA methods during the learning phase of WSVM using leave-one-day-out cross validation strategy, the reader can refer to our previous work in [58].
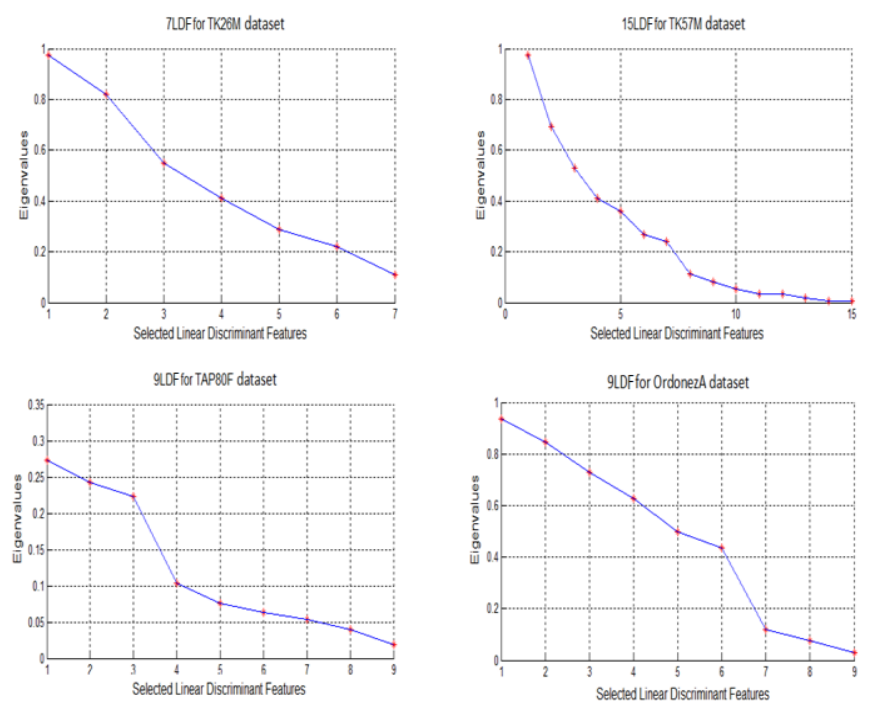

Fig. 8 Feature extraction by Linear Discriminant Analysis (LDA). 
Table 9 Recall, Precision, F-measure and Accuracy results for all approaches. Nb Feat shows the number of features PCF, LDF and PCF-LDF concatenation. Bold values are the highest F-Measure for each dataset.

\begin{tabular}{|c|c|c|c|c|c|c|c|c|}
\hline Datasets & \multicolumn{2}{|c|}{ Approaches } & Cross validation & $\mathrm{Nb}$ Feat & Recall (\%) & Precision $(\%)$ & F-Measure $(\%)$ & Accuracy \\
\hline \multirow[t]{8}{*}{ TK26M } & & HMM [2] & \multirow{8}{*}{$\begin{array}{c}\text { 28-Leave One Day } \\
\text { Out }\end{array}$} & 28 & 79.4 & 78.8 & 79.1 & 94.5 \\
\hline & & CRF [2] & & 28 & 70.8 & 74.4 & 72.5 & 95.6 \\
\hline & Models & SVM & & 28 & 61.8 & 73.3 & 67.0 & 95.5 \\
\hline & & WSVM & & 28 & 72.8 & 74.6 & 73.7 & 92.5 \\
\hline & \multirow{4}{*}{ Hybrid Models } & PCA-WSVM [58] & & 6 & 71.5 & 71.5 & 71.5 & 91.2 \\
\hline & & LDA-WSVM [58] & & 7 & 77.0 & 78.4 & 77.7 & 93.5 \\
\hline & & PCA-LDA-HMM & & 13 & 81.2 & 83.1 & 82.1 & 94.3 \\
\hline & & Proposed & & 13 & 78.8 & 80.1 & 79.4 & 95.6 \\
\hline \multirow[t]{8}{*}{ TK57M } & \multirow{4}{*}{ Models } & HMM [59] & \multirow{8}{*}{$\begin{array}{c}\text { 18-Leave One Day } \\
\text { Out }\end{array}$} & 42 & 40.0 & 37.0 & 39.0 & 76.0 \\
\hline & & CRF [59] & & 42 & 30.0 & 36.0 & 33.0 & 78.0 \\
\hline & & SVM & & 42 & 35.6 & 34.9 & 35.2 & 80.8 \\
\hline & & WSVM & & 42 & 40.8 & 37.8 & 39.2 & 77.1 \\
\hline & \multirow{4}{*}{ Hybrid Models } & PCA-WSVM [58] & & 7 & 36.5 & 34.2 & 35.3 & 76.9 \\
\hline & & LDA-WSVM [58] & & 15 & 42.3 & 39.8 & 41.0 & 77.2 \\
\hline & & PCA-LDA-HMM & & 22 & 40.9 & 44.8 & 42.8 & 73.6 \\
\hline & & Proposed & & 22 & 43.8 & 45.9 & 44.8 & 81.4 \\
\hline \multirow{8}{*}{ Tap80F } & \multirow{4}{*}{ Models } & HMM [60] & \multirow{8}{*}{$\begin{array}{c}\text { 14-Leave One Day } \\
\text { Out }\end{array}$} & 140 & 33.9 & 40.4 & 36.8 & 40.2 \\
\hline & & CRF [60] & & 140 & 27.1 & 29.5 & 28.2 & 77.2 \\
\hline & & SVM & & 140 & 15.2 & 30.0 & 20.1 & 75.6 \\
\hline & & WSVM & & 140 & 29.2 & 29.4 & 29.3 & 28.7 \\
\hline & \multirow{4}{*}{ Hybrid Models } & PCA-WSVM [58] & & 31 & 29.6 & 29.4 & 29.5 & 22.4 \\
\hline & & LDA-WSVM [58] & & 9 & 38.7 & 45.7 & 41.9 & 28.7 \\
\hline & & PCA-LDA-HMM & & 40 & 23.6 & 40.8 & 29.9 & 28.0 \\
\hline & & Proposed & & 40 & 41.4 & 49.6 & 45.1 & 75.8 \\
\hline \multirow{7}{*}{ OrdonezA } & \multirow{3}{*}{ Models } & HMM & \multirow{7}{*}{$\begin{array}{c}\text { 14-Leave One Day } \\
\text { Out }\end{array}$} & 24 & 60.4 & 62.9 & 61.6 & 63.7 \\
\hline & & SVM & & 24 & 60.0 & 58.2 & 59.1 & 85.2 \\
\hline & & WSVM & & 24 & 63.7 & 64.2 & 63.9 & 84.4 \\
\hline & \multirow[t]{4}{*}{ Hybrid Models } & PCA-WSVM & & 4 & 64.0 & 64.4 & 64.2 & 84.5 \\
\hline & & LDA-WSVM & & 9 & 65.3 & 68.9 & 67.0 & 87.1 \\
\hline & & PCA-LDA-HMM & & 13 & 65.6 & 70.4 & 67.9 & 86.5 \\
\hline & & Proposed & & 13 & 65.0 & 71.7 & 68.2 & 88.4 \\
\hline
\end{tabular}

The overall performances of our approach on the four datasets in terms of Recall, Precision, F-Measure and Accuracy, during the testing phase are summarized in Table 9 where the evaluation is averaged over all classes. A large number of experiments with HMM, CRF, SVM, WSVM, and hybrid models PCA-WSVM, LDA-WSVM and PCA-LDA-HMM is also reported. The results demonstrate that our approach outperforms other methods in terms of F-Measure for all datasets except for TK26M dataset, where it is outperformed by PCA-LDA-HMM. The results show that the combined feature set PCF-LDF contributes to significantly enhance the performance of individual classifiers, especially HMM and SVM.

The results also show that LDA outperforms PCA for recognizing activities with WSVM classifier for all datasets. One also notices that the combined method PCA-WSVM improves the classification results over CRF, SVM and WSVM classifiers only for the Tap80F dataset.

In order to detail the performance of the classifiers with respect to each individual class of daily activity recognition, we report in figures 9 and 10 the classification results in terms of accuracy measure for each activity when using HMM, WSVM, PCA-LDA-HMM and the proposed method for the TK26M and Tap80F datasets, respectively.

In TK26M dataset, PCA-LDA-HMM and the proposed approach outperform HMM and WSVM, respectively, almost in all performance metrics. Additionally, one also notices a clear superiority of PCA-LDA-HMM in recognizing the minority activities as 'Idle', 'Dinner', 'Drink', while achieving comparable results with our proposed method in the rest of activities. Nevertheless with the augmented feature space, it is 
easy to see for instance that HMM surpasses WSVM for all activities.

In Tap80F dataset, the proposed approach gives the best results for the minority activities 'Toileting', 'Take medication', 'Prep-Lunch', 'Prep-Snack', 'Washing dishes' and 'Watching TV' as opposed to other methods, while relatively lower performance to PCA-LDA-HMM for the 'Prep-Breakfast' and 'Listen music' activities. Additionally, from Fig. 9 and Fig. 10, one notices that kitchen related activities are in general harder to recognize than other activities. Such observation is still valid across all datasets employed.

In order to quantify the extent to which one class is harder to recognize than another one, we analyzed the confusion matrices of PCA-LDA-HMM and the proposed method for TK26M and Tap80F datasets (see Tables 10, 11, 12, 13), which show the accuracy results for each activity separately, where rows and columns represent the current and predicted activities, respectively. The values are provided in percentages.

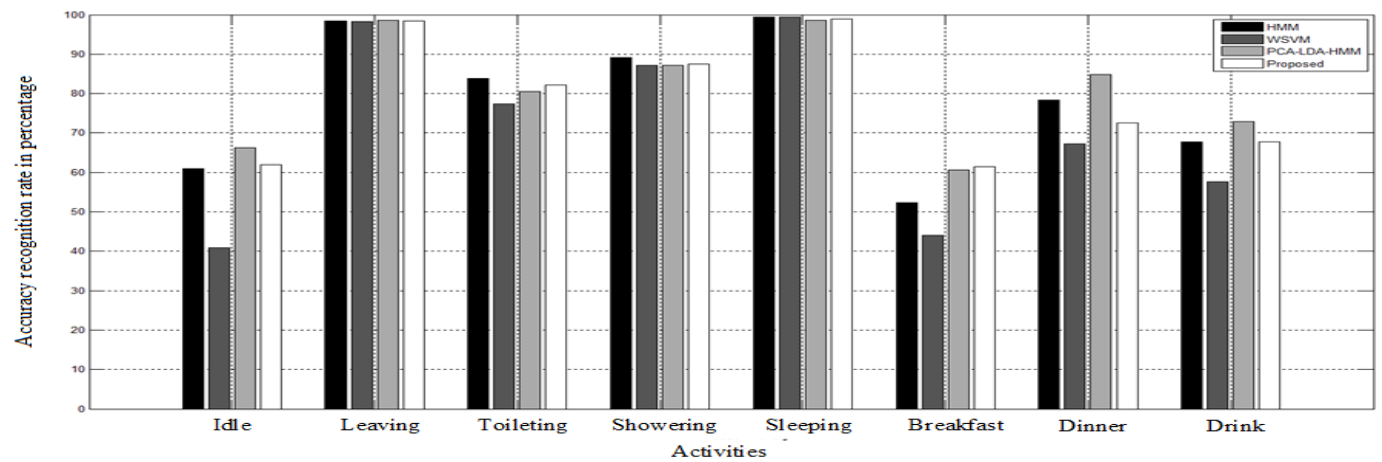

Fig. 9 Accuracy recognition rate for each activity on TK26M dataset.

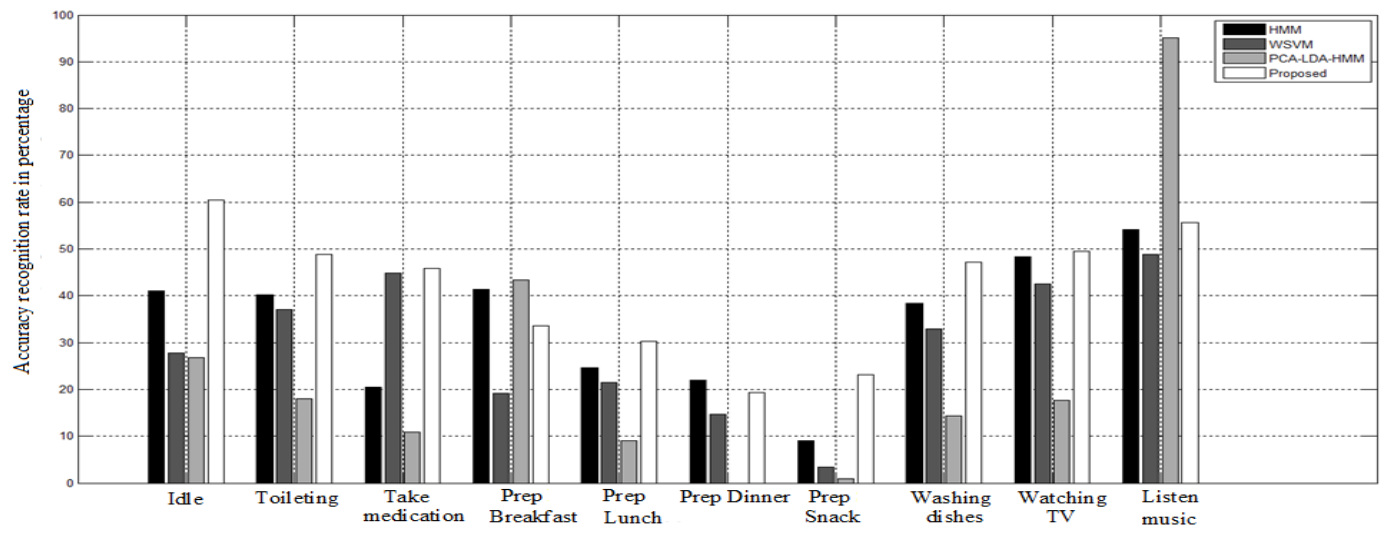

Activities

Fig. 10 Accuracy recognition rate for each activity on Tap80F dataset.

Table 10 Confusion matrix of activities for PCA-LDA-HMM method on the TK26M dataset.

\begin{tabular}{ccccccccc}
\hline Activities & Id & Le & To & Sh & Sl & Br & Di & Dr \\
\hline Id & $\mathbf{6 6 . 3}$ & 7.0 & 1.1 & 9.0 & 4.6 & 0.5 & 10.9 & 0.6 \\
Le & 0.7 & $\mathbf{9 8 . 6}$ & 0.2 & 0.2 & 0.0 & 0.1 & 0.2 & 0.0 \\
To & 9.2 & 3.2 & $\mathbf{8 0 . 5}$ & 1.6 & 5.0 & 0.0 & 0.0 & 0.5 \\
Sh & 7.5 & 0.0 & 5.3 & $\mathbf{8 7 . 2}$ & 0.0 & 0.0 & 0.0 & 0.0 \\
Sl & 0.1 & 0.0 & 0.2 & 0.1 & $\mathbf{9 8 . 6}$ & 0.0 & 0.0 & 1.0 \\
Br & 29.4 & 0.0 & 1.8 & 0.0 & 0.9 & $\mathbf{6 0 . 6}$ & 5.5 & 1.8 \\
Di & 9.8 & 0.6 & 1.4 & 0.0 & 0.0 & 2.3 & $\mathbf{8 4 . 8}$ & 1.1 \\
Dr & 11.8 & 1.7 & 1.7 & 0.0 & 0.0 & 5.1 & 6.8 & $\mathbf{7 2 . 9}$
\end{tabular}


Table 11 Confusion matrix of activities for PCA-LDA-HMM method on the Tap80F dataset.

\begin{tabular}{ccccccccccc}
\hline Activities & Id & To & T.m & P.b & P.1 & P.d & P.s & W.d & TV & L.m \\
\hline Id & $\mathbf{2 6 . 8}$ & 6.2 & 0.6 & 1.8 & 3.5 & 1.5 & 0.1 & 0.4 & 2.5 & 56.6 \\
To & 18.3 & $\mathbf{1 7 . 9}$ & 0.0 & 3.0 & 6.2 & 0.0 & 1.6 & 0.8 & 0.0 & 52.2 \\
T.m & 30.3 & 10.3 & $\mathbf{1 0 . 8}$ & 4.8 & 0.0 & 6.0 & 0.0 & 0.0 & 1.6 & 36.2 \\
P.b & 12.0 & 7.5 & 0.0 & $\mathbf{4 3 . 3}$ & 23.6 & 3.2 & 0.0 & 4.1 & 0.9 & 5.4 \\
P.1 & 3.1 & 0.0 & 0.0 & 3.3 & $\mathbf{9 . 0}$ & 7.7 & 0.0 & 2.8 & 1.1 & 73.0 \\
P.d & 0.0 & 0.0 & 0.0 & 0.0 & 0.0 & $\mathbf{0 . 0}$ & 0.0 & 0.0 & 0.0 & 100 \\
P.s & 12.5 & 0.0 & 0.0 & 2.5 & 1.6 & 0.0 & $\mathbf{0 . 9}$ & 4.4 & 0.0 & 78.1 \\
W.d & 11.3 & 6.7 & 0.0 & 1.2 & 4.0 & 0.6 & 0.0 & $\mathbf{1 4 . 3}$ & 2.1 & 59.8 \\
TV & 4.1 & 8.1 & 0.0 & 1.9 & 1.0 & 0.0 & 0.0 & 0.3 & $\mathbf{1 7 . 7}$ & 66.9 \\
L.m & 2.9 & 0.0 & 0.0 & 0.0 & 1.5 & 0.0 & 0.0 & 0.4 & 0.0 & $\mathbf{9 5 . 2}$
\end{tabular}

Table 12 Confusion matrix of activities for the proposed method on the TK26M dataset.

\begin{tabular}{ccccccccc}
\hline Activities & Id & Le & To & Sh & Sl & Br & Di & Dr \\
\hline Id & $\mathbf{6 1 . 9}$ & 6.4 & 0.6 & 9.8 & 4.6 & 0.9 & 14.7 & 1.1 \\
Le & 0.8 & $\mathbf{9 8 . 4}$ & 0.2 & 0.3 & 0.0 & 0.1 & 0.1 & 0.0 \\
To & 10.0 & 2.9 & $\mathbf{8 2 . 2}$ & 2.1 & 2.1 & 0.5 & 0.2 & 0.0 \\
Sh & 7.1 & 0.0 & 4.5 & $\mathbf{8 7 . 5}$ & 0.0 & 0.1 & 0.5 & 0.3 \\
Sl & 0.1 & 0.5 & 0.4 & 0.1 & $\mathbf{9 8 . 9}$ & 0.0 & 0.0 & 0.0 \\
Br & 20.9 & 0.0 & 0.9 & 0.0 & 0.4 & $\mathbf{6 1 . 4}$ & 8.2 & 8.2 \\
Di & 18.4 & 0.8 & 0.3 & 0.0 & 0.3 & 3.1 & $\mathbf{7 2 . 5}$ & 4.6 \\
Dr & 18.7 & 1.7 & 0.0 & 0.0 & 0.0 & 8.4 & 3.4 & $\mathbf{6 7 . 8}$
\end{tabular}


Table 13 Confusion matrix of activities for the proposed method on the Tap80F dataset.

\begin{tabular}{ccccccccccc}
\hline Activities & Id & To & T.m & P.b & P.1 & P.d & P.s & W.d & TV & L.m \\
\hline Id & $\mathbf{6 0 . 4}$ & 6.4 & 9.6 & 4.3 & 5.2 & 1.1 & 2.5 & 1.5 & 5.3 & 3.7 \\
To & 14.7 & $\mathbf{4 8 . 9}$ & 7.5 & 3.3 & 8.7 & 1.1 & 5.1 & 5.8 & 3.5 & 1.4 \\
T.m & 26.5 & 10.3 & $\mathbf{4 5 . 9}$ & 5.9 & 0.0 & 2.6 & 3.1 & 4.1 & 1.6 & 0.0 \\
P.b & 13.4 & 3.8 & 11.2 & $\mathbf{3 3 . 6}$ & 24.8 & 3.2 & 1.9 & 4.7 & 3.2 & 0.2 \\
P.1 & 14.1 & 2.8 & 13.7 & 13.9 & $\mathbf{3 0 . 3}$ & 5.9 & 3.2 & 7.8 & 3.4 & 4.9 \\
P.d & 10.7 & 3.1 & 30.0 & 6.2 & 16.8 & $\mathbf{1 9 . 3}$ & 3.9 & 7.9 & 1.4 & 0.7 \\
P.s & 11.5 & 5.9 & 21.5 & 8.7 & 10.9 & 7.2 & $\mathbf{2 3 . 1}$ & 5.6 & 1.9 & 3.7 \\
W.d & 14.0 & 3.9 & 7.3 & 3.9 & 9.8 & 3.3 & 2.4 & $\mathbf{4 7 . 2}$ & 2.7 & 5.5 \\
TV & 17.6 & 10.6 & 1.0 & 0.7 & 1.5 & 0.8 & 0.8 & 17.5 & $\mathbf{4 9 . 5}$ & 0.0 \\
L.m & 15.7 & 4.7 & 4.7 & 1.3 & 6.6 & 1.3 & 1.6 & 6.6 & 1.9 & $\mathbf{5 5 . 6}$
\end{tabular}

From tables 10 and 12, one notices that the activities 'Leaving', 'Toileting', 'Showering', 'Sleeping' and 'Dinner' are better recognized comparatively with 'Idle' and the two kitchen activities 'Breakfast', and 'Drink'. The 'Dinner' activity using our method seems to be less recognized compared to PCA-LDA-HMM. In Table 12, $61 \%$ of 'Prep.Breakfast' activity instances are correctly recognized, while $21 \%$ goes into 'Idle' and $16 \%$ are confused with Prepare meal activities. The other similar classes such as 'Dinner' and 'Drink' show similar trend of sharing errors among each other. We notice that the 'Breakfast' and 'Dinner' activities share $10 \%$ of their instances among each other due to being performed at the same location.

In Table 11, for the Tap80F dataset, only the "listen music" activity has been successfully recognized (with recognition rate of $95 \%$ ) compared to other activities. The results also show that 'Prep.dinner' and 'Prep.snack' have not been recognized. In Table 13, the kitchen activities: 'Prep.Breakfast', 'Prep.Lunch', 'Prep.Dinner' and 'Prep.Snack', do not perform well in comparison to other activities. In 'Take medication' activity, $46 \%$ of activity instances are accurately assigned labels, while $26 \%$ of instances confused with 'Idle' activity and $11 \%$ with Prepare meal activities. Intuitively, this can be explained by the fact that 'Take medication' activity is performed during the meal preparation activities.

Strictly speaking, the relatively reduced performance of our approach at few activity recognition tasks testifies of the still ongoing challenges raised by such complex tasks, and the difficulty of designing a universal classifier that outperforms alternatives at each individual class. Nevertheless, when taking the average result across all activity classes, the superiority of the proposed approach cannot be ignored as can be seen from Table 9 .

\subsection{Discussion}

Throughout this study, one shall point out several interesting notes:

- The experimental results shown in previous section demonstrate that the proposed approach consistently outperforms the other approaches in correct assignment of activity instances to the target class in the case of limited number of instances of the minority classes. This work has been argued that it is extremely important to use class information in feature extraction for supervised learning. It can be shown in our results that LDA-WSVM outperforms PCA-WSVM for all datasets. However, LDA method, although using class information, suffers from reduced performance due to its parametric nature. This explains the discriminating power of combined method using PCA-LDA. We also noticed that PCA method seems appropriate for the extraction of features in the datasets with large features vectors as in Tap80F dataset.

- The use of the modified weighted multi-SVM can effectively handle the traditional algorithm's duplicate training data, the impact of noise points and the training scale. At the same time, it compensates for the impact of different sizes of training data, reduces the training error, and improves the classification accuracy of the traditional SVM particularly for small samples. This method is shown to be significantly more effective than standard SVM and CRF methods for all datasets and outperforms the "reference" HMM method. Besides, CRF and SVM are known to be sensitive to overfitting on a dominant class than other 
methods

- HMM outperforms CRF, SVM and WSVM for TK26M and Tap80F datasets. HMM is trained by splitting the training data in which a separate model $P(x \mid y)$ is learned for each class. This partly explains why HMM performs better in the minority activities.

- Although, HMM and modified Weighted SVM are efficient compared to other methods for all datasets in overall, by using both the PCF and LDF in a concatenated feature matrix, we achieved the highest performance of classification for both HMM and WSVM.

- A close look at the employed dataset shows an important discrepancy in terms of activities embedded. For instance, TK57M contains (almost) only half of class activities contained in TK26M. Besides, only 'Idle', 'Breakfast' and 'Toileting' activities are present in all datasets, while other activities only occur in one single dataset (e.g., 'Washing dishes', 'Listening to music'). The independence of the tasks can also be questioned. For instance the task eating is ultimately linked to activity 'Lunch' and 'Dinner' occurring in TK57M. Therefore, the generalization of the results of one classifier from one dataset to another should be taken with cautious.

- Given the success of deep-learning like methodologies in other disciplines, e.g., computer vision, text mining, one may also question whether such techniques can enhance the daily activity recognition in our case. Although a comprehensive answer to such question deserves several studies, one shall mention the following. First, the application of deep learning like approaches to daily activity recognition is very much premature and only tinny proportions were reported. Second, the few technical papers reported in the literature in the field were mainly concerned by the optimization of the feature selection process in order to maximize the individual class recognition rate. Consequently, when only a small number of features is permissible due to environmental constraints, a situation which applies to our Benchmark dataset, the application of such approach is debatable. Third, the existence of wide range of deep learning methods in literature makes the choice of relevant method debatable. Nevertheless, this opens the door to further hybridation scenarios between standard like machine learning approaches and deep learning.

- From data gathering perspective, we suspect that the use of a the Bluetooth headset for annotating data as in house TK26M yields more accurate results than using other annotation methods. Indeed, a Bluetooth headset was used for data transmission across the sensor network. This means that the timestamps of the annotation were synchronized with the timestamps of the sensors. In the TK57M and OrdonezA datasets, the activity diaries were used, which makes it more error prone because the timestamps might not always be written down correctly and the diaries have to be typed over afterwards. However, the results obtained with OrdonezA are better compared to TK57M for all recognition methods because this house includes more activity classes. In the Tap80F dataset, activities were annotated using a PDA system. Sometimes subjects specified one activity and carried out a different activity. Also the user can selected the wrong activity from the list by mistake.

- Given the considerations pointed out previously, the high performance obtained in the case of TK26M dataset, which seems to be less vulnerable to classoverlapping than others, as compared to other datasets. This overlapping between the activities is due to the layout of the house. In the TK26M House, there is a separate room for almost every activity. From this dataset, the 'Idle', 'Breakfast', 'Dinner' and 'Drink' yield the worst results compared to the others activities. Most confusion occurs between the 'Idle' activity and the three kitchen activities. In particular, 'Idle' is one of the most frequent activities in all datasets but is usually not a very important activity to recognize, and makes the dataset particularly hard. It might therefore be preferable to accept loosing accuracy on this activity if it allows a better recognition of minority classes. The kitchen activities are food related tasks, they are also very challenging tasks to be recognized because most of the instances of these activities were performed in the same location sharing the same set of sensors, which result in less inter-class variations. The activities 'Toileting' and 'Showering' are more separable because they are in two different locations in TK26M house. This means for most activities the door sensor to the room the activity is performed in is very informative. Therefore the location of the sensors is of great importance for the performance of the recognition system.

\section{Conclusions and Future work}

In this paper, a new PCA-LDA-WSVM scheme has been proposed to recognize ADL from smart home environments. This approach makes use of "Change point" and "Last" representation of binary data issued from a sensor network. The method shows three merits. First, after feature extraction by PCA and LDA, and restricted to the most significant principal components and linear discriminants features, the training set is reduced, and the prediction accuracy is improved. Second, the modified Weighted SVM classifier setting different cost parameters for each activity was employed in order to handle the imbalanced human activity datasets and shows significant improvement as compared to SVM (with equal costs). Third, from the detailed analysis and comparisons of results, it can be concluded that compared to existing approaches the proposed learning method proves to be more effective and reliable in correct classification of activities in the case of 
limited amount of training data, variations in activity patterns and frequencies of execution of activities. Furthermore, we observed that differences in the layout of houses and the way a dataset was annotated can greatly affect the performance in activity recognition. The work presented here demonstrates that a suitable sequence feature set combined with the modified WSVM based our criterion classifier achieve good improvement and efficiency over the traditional used methods.

As perspective work, one shall point the growing interest in deep learning methodologies in machine learning community, therefore, a hybridation of SVM with an appropriate class of deep learning like approach is promising.

\section{REFERENCES}

1. Tapia E. M, Intille S. S, \& Larson K. Activity recognition in the home using simple and ubiquitous sensors, Springer Berlin / Heidelberg, vol. 3001, pp. 158-175, 2004.

2. Van Kasteren T, Noulas A, Englebienne G, \& Kröse B. Accurate activity recognition in a home setting, in Proceedings of UbiComp'08. New York, NY, USA: ACM, 2008, pp. 1-9.

3. Ordóñez F.J, de Toledo P, Sanchis A. Activity Recognition Using Hybrid Generative/Discriminative Models on Home Environments Using Binary Sensors. Sensors 2013, 13, 5460-5477.

4. Logan B, Healey J, Philipose M, Tapia E. M, and Intille S. A Long-Term Evaluation of Sensing Modalities for Activity Recognition. In: Proceedings of the $9^{\text {th }}$ International Conference on Ubiquitous Computing, 2007, Springer Berlin Heidelberg,pp. 483-500.

5. Chen L, Hoey J, Nugent C, Cook D and Yu Z, Sensor based activity recognition, IEEE Transactions on Systems, Mans and Cybernetics, Part C, 42(6), 2012, p. 790-808

6. Kidd C. D, Orr R, Abowd G. D, Atkeson C. G, Essa I. A, MacIntyre B, Mynatt E. D, Starner T, and Newstetter W. The aware home: A living laboratory for ubiquitous computing research. In Proceedings of the Second International Workshop on Cooperative Buildings, Integrating Information, Organization, and Architecture, CoBuild '99, pages 191- 198, London, UK, UK, 1999. Springer-Verlag

7. Kasteren T. L. M, Englebienne G, and Krose B. J. A. Human activity recognition from wireless sensor network data: Benchmark and software. In Activity Recognition in Pervasive Intelligent Environments, $\mathrm{p}$ 165-186. Atlantis Press, Paris, France, 2011

8. Cook D. J, Augusto J. C, and Jakkula V. R. Ambient intelligence: Technologies, applications, and opportunities. Pervasive and Mobile Computing, 5(4):277 - 298, 2009.
9. Barger T, Alwan M, Kell S, Turner B, Wood S, and Naidu A. Objective remote assessment of activities of daily living: Analysis of meal preparation patterns. Poster presentation, Medical Automation Research Center, University of Virginia Health System, 2002

10. Cook D, Schmitter-Edgecombe M, Crandall A, Sanders $\mathrm{C}$, and Thomas B. Collecting and disseminating smart home sensor data in the casas project. In Proc. of CHI09 Workshop on Developing Shared Home Behavior Datasets to Advance HCI and Ubiquitous Computing Research, 2009

11. Oppurtunity EU project, http://www.opportunityproject.eu/node/58 (accessed on April, 2015)

12. http://research.nokia.com/page/12000 (accessed April 2015)

13. The Aware Home, http://awarehome.imtc.gatech.edu (accessed April 2015)

14. http://domus.usherbrooke.ca/jeux-de-donnees/ (accessed April 2015)

15. Aggrawal J. K and Ryoo M. S, Human activity analysis: A review, ACM Computing Surveys, 43(3), 2011, p. $16: 1-16: 43$

16. Krishnan N. C, Cook D. J, Activitv Recognition on Streaming Sensor Data. Pervasive and Mobile Computing, 10, 2014, p 138-154

17. Andreu J, Angelov P. Real-time human activity recognition from wireless sensors using evolving fuzzy systems, in Proc. IEEE World Congress on Computational Intelligence, 2010, pp 2652-2659.

18. Chen L, Nugent C. D, and Wang H. A knowledgedriven approach to activity recognition in smart homes. IEEE Transactions on Knowledge and Data Engineering, 24:961-974, 2012.

19. Bishop C, Pattern Recognition and Machine Learning, Springer. New York, ISBN: 978-0-387-31073-2, 2006.

20. Huang W, Zhang J, \& Liu Z. Activity recognition based on hidden Markov models. In Knowledge Science, Engineering and Management (pp. 532-537). Springer Berlin Heidelberg (2007).

21. Vail DL, Veloso MM, Lafferty JD, Conditional random fields for activity recognition. In: International conference on autonomous agents and multi-agent systems (AAMAS), 2007.

22. Abidine M.B and Fergani B. Evaluating C-SVM, CRF and LDA classification for daily activity recognition, in Proc. of IEEE ICMCS, Tangier-Morocco, pp. 272 -277, IEEE, 2012.

23. Liu S, Gao R.X, John D, Staudenmayer J, Freedson P.S: Classification of physical activities based on sparse representation. In: 2012 Annual International Conference of the IEEE Engineering in Medicine and Biology Society (EMBC), August 28-September 1, pp. 6200-6203 (2012).

24. Fang H, He L: BP Neural Network for Human Activity Recognition in Smart Home. In: 2012 International Conference on Computer Science \& Service System(CSSS), August 11-13, pp. 1034-1037 (2012).

25. Fleury A, Vacher M, Noury N, "SVM-Based MultiModal Classification of Activities of Daily Living in Health Smart Homes : Sensors, Algorithms and First 
Experimental Results," IEEE Transactions on Information Technology in Biomedicine, Vol. 14(2), pp. 274-283, March 2010.

26. Abidine M.B, Fergani B, Clavier L. ImportanceWeighted the Imbalanced data for $C$-SVM Classifier to Human Activity Recognition. Proc. of IEEE WOSSPA'13, 2013, pp. 330-335.

27. Goodfellow I., Bengio Y., and Courville A., Deep Learning, 2016. MIT Press

28. Ordóñez F. J., and Roggen D., Deep Convolutional and LSTM Recurrent Neural Networks for Multimodal Wearable Activity Recognition, Sensors, 16(115), 2016, p. 1-25.

29. Plotz T., Hammerla N.Y., Olivier P., Feature learning for activity recognition in ubiquitous computing, In IJCAI Proceedings-International Joint Conference on Artificial Intelligence (Vol. 22, No. 1, p. 1729-1735), 2011.

30. Pechenizkiy M, Tsymbal A, Puuronen S. 2005. On Combining Principal Components with Parametric LDA-based Feature Extraction for Supervised Learning. In: T.Morzy et al. (Eds.), Proc. of $1^{\text {st }}$ Int. Workshop on Data Mining and Knowledge Discovery, ADMKD'05, Estonia, pp. 47-56.

31. Guyon I, Gunn S, Nikravesh M, and Zadeh L. A, "Feature Extraction: Foundations and Applications," Berlin, Heidelberg: Springer-Verlag, 2006.

32. Chawla N. V. Data mining for imbalanced datasets: An overview. In Data Mining and Knowledge Discovery Handbook,(pp. 875-886). Springer US, 2010.

33. Akbani R, Kwek S, Japkowicz N. Applying support vector machines to imbalanced datasets. In Machine Learning: ECML 2004 (pp. 39-50). Springer Berlin Heidelberg.

34. Hulse J.V, Khoshgoftaar T.M and Napolitano A. "Experimental perspectives on learning from imbalanced data," In Proc. of the $24^{\text {th }}$ International Conference on Machine Learning, Corvallis, OR, 2007, pp.143-146.

35. Wu G, and Chang E. Y, KBA: Kernel boundary alignment considering imbalanced data distribution, IEEE Transactions on Knowledge and Data Engineering, vol. 17, no. 6, pp. 786-795, 2005.

36. Raskutti B and Kowalczyk A, Extreme re-balancing for SVMs: a case study, SIGKDD Explorations, vol. 6, no. 1, pp. 60-69, 2004.

37. Huang Y. M, \& Du S.X. Weighted support vector machine for classification with uneven training class sizes, In: Proceedings of the IEEE International Conference on Machine Learning and Cybernetics. 2005, Vol. 7, pp. 4365-4369.

38. Abidine, M.B., Fergani, L., Fergani, B., Fleury, A.: Improving human activity recognition in smart homes. International Journal of E-Health and Medical Communications (IJEHMC) 6(3), 19-37 (2015).

39. Frank A and Asuncion A. UCI machine learning repository, 2010.
40. Datasets for Activity Recognition, http://sites.google.com/site/tim0306/ (accessed February 09, 2012)

41. http://courses.media.mit.edu/2004fall/mas622j/04.projec ts/home/ (accessed January, 2013)

42. http://mlr.cs.umass.edu/ml/datasets/Activities+of+Daily +Living+\%28ADLs\%29+Recognition+Using+Binary+S ensors (accessed April , 2015)

43. Jolliffe I. T. (2010). Principal component analysis (2nd ed.).NewYork, NY: Springer-Verlag.

44. Peterek, T., Penhaker, M., Gajdoš, P., and Dohnálek, P. "Comparison of classification algorithms for physical activity recognition." In Innovations in Bio-inspired Computing and Applications, pp. 123-131. Springer International Publishing, 2014

45. Duda R. O., Hart P. E., and Stork D. G., Pattern classification. John Willy and sons, Inc., 2001

46. Dargham J. A, Chekima A, Moung E. G, Fusion of PCA and LDA Based Face Recognition System, International Conference on Software and Computer Applications (ICSCA2012), vol. 41, pp.111-116.

47. Marcialis G. L. and Roli F, Fusion of LDA and PCA for Face Verification, in Proceedings of the Workshop on Machine Vision and Perception, 8th Workshop of the Italian Association for Artificial Intelligence (ALLA 02), 2002.

48. Cortes C and Vapnik V, Support vector network, Machine Learning 20, 1995, p. 1-25

49. Osuna E, Freund R, Girosi F. "Support vector machines: training and applications". Massachusetts Institute of Technology, Cambridge, MA, USA, 1997.

50. Veropoulos K, Campbell $\mathrm{C}$ and Cristianini N, "Controlling the sensitivity of support vector machines", Proceedings of the International Joint Conference on AI, 1999, pp. 55-60.

51. Hsu C.W, Chang C.C, Lin C.J: A Practical Guide to Support Vector Classification, (2008), available at http://www.csie.ntu.edu.tw/ cjlin/papers/guide/guide.pd $\mathrm{f}$

52. Chathuramali M, Rodrigo R, Faster human activity recognition with SVM, in: Proceedings of International Conference of Advances in ICT for Emerging Regions, IEEE, 2012, 197-203. Rabiner LR (1989) A tutorial on hidden markov models and selected applications in speech recognition. Proc IEEE 77(2):257-286

53. Rabiner L. R. A tutorial on hidden markov models and selected applications in speech recognition. Proceedings of the IEEE, 77(2):257-286, 1989.

54. C. J. C. Burges, "A tutorial on support vector machines for pattern recognition," Data Mining and Knowledge Discovery, vol. 2, no. 2, pp. 121 - 167, 1998.

55. Chang CC, Lin CJ, (2011) LIBSVM: a library for support vectormachines. ACM Trans Intell Syst Technol 2:1-27, [Online]. Available: http://www.csie.ntu.edu.tw/ cjlin/libsvm/ 
56. Chen P.H, Lin C.J, and Scholköpf B. A tutorial on $n u-$ Support Vector Machines. Applied Stochastic Models in Business and Industry, 21:111-136, 2005.

57. Katz, S., Down, T.D., and Cash, H.R. Progress in the development of the index of adl. Gerontologist, (1970) 20-30.

58. Abidine M.B, Fergani B, News Schemes for Activity Recognition Systems Using PCA-WSVM, ICA-WSVM, and LDA-WSVM. Information, 6(3), 505-521 (2015)

59. Van Kasteren T, Alemdar H and Ersoy C. Effective Performance Metrics for Evaluating Activity Recognition Methods. In Proceedings of the ARCS 2011 - 24th International Conference on Architecture of Computing Systems, Comot, Italy, 24-25 February 2011; p. 10.

60. Van Kasteren T, Englebienne G, Kröse B.J: An activity monitoring system for elderly care using generative and discriminative models. Personal Ubiquitous Comput. 14, 489-498 (2010). 